\title{
Vibration Analysis of Viscoelastic FGM Nanoscale Plate Resting on Viscoelastic Medium Using Higher-order Theory
}

\author{
Hamzi Souad ${ }^{1}$, Mechab Ismail ${ }^{2}$, Abbad Hichem $^{3}$, Elmeiche Noureddine ${ }^{3 *}$ \\ ${ }^{1}$ Laboratory of Sciences and Water Techniques (LSTE), Department of Civil Engineering, Faculty of Science and Technology, Mustapha \\ Stambouli University of Mascara, Avenue Cheikh El Khaldi, 29000 Mascara, Algeria \\ ${ }^{2}$ Laboratory of Mechanics and Physics of Materials (LMPM), Department of Mechanical Engineering, Faculty of Technology, Djilali \\ Liabes University, BP89 Avenue ben M'hidi, 22000 Sidi Bel-Abbes, Algeria \\ ${ }^{3}$ Civil and Environmental Engineering Laboratory (LGCE), Department of Civil Engineering and publics' works, Faculty of Technology, \\ Djilali Liabes University, BP89 Avenue ben M'hidi, 22000 Sidi Bel-Abbes, Algeria \\ * Corresponding author, e-mail: noureddine.elmeiche@univ-sba.dz
}

Received: 23 March 2020, Accepted: 05 October 2020, Published online: 05 November 2020

\begin{abstract}
The present article aims essentially to present an analytical and numerical method which makes it possible to study the damped vibrations of viscoelastic FGM nanoplates resting on viscoelastic foundations. A new model for the higher-order shear deformation plate theory is coupled with the internal Kelvin - Voigt viscoelastic model and the three-parameter viscoelastic foundation model for the purpose of reducing and minimizing the vibration response of the system. It is widely admitted that the mechanical properties of these new functionally gradient materials (FGMs) vary according to the thickness of the plate and depend on its volume fraction. The use of FGM plates seems to be an ideal solution for the study of free vibrations because of their multifunctionality that is fully integrated with the nonlocal Eringen effect. The dynamic response of such a complex system has been investigated by varying the aspect ratio of the plate, the mechanical characteristics of the material used, the internal and external damping and the foundation rigidity. The results obtained, with and without the nonlocal effect, were compared with those of different models of higher-order theories and under various boundary conditions; they were found to be in good agreement with those reported in the literature.
\end{abstract}

\section{Keywords}

higher-order plate theory, FGM materials, viscoelasticity, nonlocal theory, Winkler-Pasternak viscoelastic foundation

\section{Introduction}

Functionally graded materials (FGMs) were used for the first time by Japanese scientists in the 1980s as high temperature-resistant materials in the area of aerospace construction. Recently, these new materials have been employed in various electrical devices, energy transformation, biomedical technology, and optical systems [1-6]. It is worth indicating that different plate configurations exist today; they are often classified according to their geometry, type of stress experienced, and type of behavior (membrane-flexion), with or without transverse shearing. The plates whose transverse shearing is neglected are called Love-Kirchhoff plates [7]. Love-Kirchhoff's theory applies to thin plates. On the other hand, thick homogeneous plates, for which shear is taken into account, are called Reissner-Mindlin plates [8-9]. The Reissner-Mindlin theory, also referred to as the first-order shear deformation theory (FSDT), is well suited for the analysis of problems linked to bending and vibration of structures. The first-order shear deformation theory (FSDT) of Reissner - Mindlin is more precise than the classical plate theory (CPT) of Love-Kirchhoff. However, Reissner Mindlin's theory requires a shear correction factor and gives a constant distribution of shear stresses across the thickness of the plate, which is not the case here. In order to represent the kinematics of a point of a beam or plate, without the shear correction factor, some higher order shear deformation theories (HSDTs) have been presented in order to describe the behavior of beams and plates under various mechanical loadings. Thus, Levinson [10] and Reddy [11] developed higher-order functions, like the higher-order shear deformation plate theory (HSDT), in terms of thickness in the form of a third-degree polynomial. In ddition, it should be noted that the variation of shear 
stress can be represented using a second degree polynomial as a function of the thickness. Several researchers, such as Touratier [12], Karama et al. [13], Aydogdu [14], Soldatos and Timarci [15], Mechab et al. [16] and Benyamina et al. [17] have developed other types of sinusoidal, hyperbolic or exponential form functions for the mechanical analysis of structures. In order to deal with the problems of static and dynamic analysis of orthotropic plates, Shimpi and Patel [18-19] developed a refined plate theory (RPT) that is classified among higher-order theories. Unlike the first-order shear deformation theory (FSDT) and higher-order shear deformation theory (HSDT), the refined plate theory reduces the calculation time and gives four equilibrium equations instead of five, without correction factor, and with a parabolic variation of shear stress through thickness of the plate. The RPT theory has recently attracted a lot of interest among researchers to solve the problem of vibrations, buckling of isotropic, orthotropic and FGM structures under various loadings [20-22].

Over the last few years, due to the rapid development of technology, particularly in the field of nanostructures that have superior mechanical properties and a large number of applications in technology, the researchers were urged to take into account the effects of scales and atomic forces in order to obtain solutions with acceptable accuracy. Eringen's nonlocal theory is based on this hypothesis which suggests that the stresses at a reference point in the body depend not only on the deformations at that point but also on the deformations at all other points of the body. Consequently, the analysis of nanostructural vibrations has become a subject of major interest for current and future research studies [23-27].

Recently, the nonlocal theory has been used in the analysis of nanobeams and nanoplates made of functionally graded materials (FGMs). For this, Farzad et al. [28] investigated the buckling of FGM nanoplates, subjected to variable thermal, linear and nonlinear loads, resting on a Pasternak-type foundation. They found out that the responses of buckling with the nonlocal effect are weaker than those with a local effect, under various loading conditions. As for Zenkour and Arefi [29], he conducted a static and dynamic analysis of an FGM nanoplate resting on a visco-Pasternak foundation, and subjected to thermo-electromechanical loading. Note that both Eringen's nonlocal elasticity theory and the classical plate theory are used for the determination of the equilibrium equations. The refined higher-order shear deformation theory was used by Żur et al. [30] for the analysis of the vibrations and buckling of FGM structures in terms of the nonlocal parameter, volume fraction index, power law index, mechanical, electrical and magnetic loads, mechanical, electrical, and magnetic loadings, as well as the geometric ratio of the section.

Viscoelastic materials are used in various fields of engineering such as the design of household appliances, automobile, aeronautics and even the vast area of civil engineering. Reducing mechanical vibrations and noise is one of the major concerns in the automotive, naval and aeronautical industries. To remedy this problem, there are anti-vibration sheets, called sandwich sheets, made of a thin layer of viscoelastic material interposed between two steel sheets. The damping capacity can therefore be improved by the viscoelastic material.

On the other hand, Wang and Tsai [31] used the finite element method (FEM) to analyze the quasi-static and dynamic response of the linear viscoelastic plate, where the temperature field is assumed to be constant and homogeneous; here, the relaxation modulus is supposed to be in the Prony series form. Kiasat et al. [32], Pouresmaeeli et al. [33], Liu et al. [34] and Hosseini et al. [35] studied the free vibrations of thin plates made of functionally graded materials and composite materials, using the Love-Kirchhoff theory, also known as the classical plate theory (CPT), resting on visco-Winkler and visco-Winkler-Pasternak foundations, using the Kelvin-Voigt viscoelastic model. As for Ebrahimi and Barati [36] and Arefi and Zenkour [37], they used a refined higher-order plate theory with a trigonometric shear stress function for the purpose of exploring the influence of viscoelastic parameters, due to hygrothermal and piezoelectric charges, on the vibration frequency of FGM nanoplates and viscoelastic sandwich nanoplates with nonlocal effect.

The present work focuses on the viscoelastic study of nanoplates and Winkler-Pasternak type foundations in order to analyze free plate vibrations using the higher-order plate theory with nonlocal effect, as well as a new shape function for the shear-stress distribution through the nanoplate thickness. It is useful to mention that the mechanical properties of the plate vary gradually through its thickness, in accordance with the distribution of the power-law FGM (P FGM). The scale effect, shear deformation, mechanical properties, damping and rigidities of the foundations are taken into account while studying the response of the structure. The results obtained for free vibrations were compared with those of different versions of higher order theories, and under various boundary conditions. 


\section{Mathematical development}

Consider a nanoplate made of FG viscoelastic materials, with length $\mathrm{a}$, width $\mathrm{b}$ and thickness $h$. The properties of the elastic materials of the FGM plate are the Young's modulus $E(z)$ and mass density $\rho(z)$. The plate rests on a viscoelastic foundation; its coordinates are illustrated in Fig. 1.

The Kelvin-Voigt model used consists of an infinite set of springs and dampers connected in parallel; the spring stiffness and damping coefficient are defined respectively by $k_{w}, k_{p}$ and $c_{d}$. The displacements of any point on the nanoplate can be expressed in terms of average displacement components of the surface. The displacement field is given by:

$$
\begin{aligned}
& U(x, y, z, t)=u_{0}(x, y, t)-z \frac{\partial w_{b}(x, y, t)}{\partial x}+(f(z)-z) \frac{\partial w_{s}(x, y, t)}{\partial x} \\
& V(x, y, z, t)=v_{0}(x, y, t)-z \frac{\partial w_{b}(x, y, t)}{\partial y}+(f(z)-z) \frac{\partial w_{s}(x, y, t)}{\partial y} \\
& W(x, y, z, t)=w_{b}(x, y, t)+w_{s}(x, y, t) .
\end{aligned}
$$

Note that $U, V$ and $W$ are the displacement components along $x, y$ and $z$, respectively. The fundamental unknowns consist of the four generalized displacements $u_{0}, v_{0}, w_{b}$, and $w_{s}$ which are functions of the coordinates $x$ and $y$. Note that $u_{0}$ and $v_{0}$ are the displacement components along $x$ and $y$, and $w_{b}$ and $w_{s}$ are the displacement components along $z$. A new transverse shear deformation shape function is given by the following expression:

$$
f(z)=\frac{1}{1-\pi \cdot e^{-\frac{1}{4}}}\left(z-h \cdot e^{\left(\frac{z}{h}\right)^{2}-\frac{1}{4}} \cdot \sin \left(\frac{z}{h} \pi\right)\right) .
$$

Different higher-order shear deformation plate theories are summarized in Table 1. According to Soldatos and Timarci [15], the shape function must meet the following three conditions:

Their derivatives should be equal to zero at the point $(x, y, \pm h / 2)$, on the top and bottom surfaces of the plate (Fig. 2(a)).

$$
\left.f^{\prime}(z)\right|_{z= \pm \frac{h}{2}}=0
$$

The function $f(z)$ must be an odd function (Fig. 2(b)).

$$
\int_{z=-h / 2}^{z=h / 2} f(z) d z=0
$$

The deformation field is expressed in Cartesian coordinates. Taking into account the warping of the straight section of the plate, the refined theory of thick plates can be written in the following form:

\begin{tabular}{|c|c|c|c|c|}
\hline Theory & & Shape function $f(z)$ & & $\begin{array}{c}\text { unknown } \\
\text { generalized } \\
\text { displacements }\end{array}$ \\
\hline $\begin{array}{l}\text { CPT(classical } \\
\text { plate theory) }\end{array}$ & & 0 & & - \\
\hline $\begin{array}{l}\text { FSDT( first } \\
\text { order theory) }\end{array}$ & & $z$ & & - \\
\hline $\begin{array}{l}\text { TSDT } \\
\text { Aghababaei } \\
\text { and Reddy [25] }\end{array}$ & & $z\left(1-\frac{4 z^{2}}{3 h^{2}}\right)$ & & $\begin{array}{c}u_{0}, v_{0}, w_{0} \\
\theta_{x}, \theta_{y}\end{array}$ \\
\hline $\begin{array}{l}\text { SSDT } \\
\text { Touratier [12] }\end{array}$ & & $\frac{h}{\pi} \sin \left(\frac{\pi z}{h}\right)$ & & $\begin{array}{c}u_{0}, v_{0}, w_{0} \\
\theta_{x}, \theta_{y}\end{array}$ \\
\hline $\begin{array}{l}\text { Present } \\
\text { model }\end{array}$ & $\frac{1}{1-\pi e^{-\frac{1}{4}}}$ & $z-h e^{\left(\frac{2}{h}\right)-\frac{1}{4}} \sin \left(\frac{z}{h} \pi\right)$ & $-z$ & $u_{0}, v_{0}, w_{b}, w_{s}$ \\
\hline
\end{tabular}

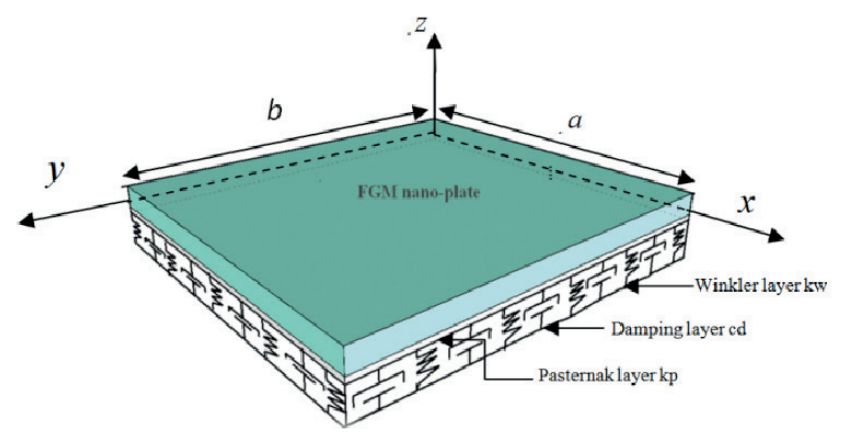

Fig. 1 Geometry of a viscoelastic FG nano-plate resting on a viscoelastic Pasternak foundation

Table 1 The various transverse shear functions used in different plate theories

$$
\begin{aligned}
& \left\{\begin{array}{c}
\varepsilon_{x x} \\
\varepsilon_{y y} \\
\gamma_{x y}
\end{array}\right\}=\left\{\begin{array}{c}
\frac{\partial u}{\partial x} \\
\frac{\partial v}{\partial y} \\
\frac{\partial u}{\partial y}+\frac{\partial v}{\partial x}
\end{array}\right\}-z\left\{\begin{array}{c}
\frac{\partial^{2} w_{b}}{\partial x^{2}} \\
\frac{\partial^{2} w_{b}}{\partial y^{2}} \\
2 \frac{\partial^{2} w_{b}}{\partial x \partial y}
\end{array}\right\}+f(z)\left\{\begin{array}{c}
\frac{\partial^{2} w_{s}}{\partial x^{2}} \\
\frac{\partial^{2} w_{s}}{\partial y^{2}} \\
2 \frac{\partial^{2} w_{s}}{\partial x \partial y}
\end{array}\right\}, \\
& \varepsilon_{z}=0,\left\{\begin{array}{l}
\frac{\partial w_{s}}{\partial x} \\
\gamma_{x z}
\end{array}\right\}=\frac{\partial f(z)}{\partial z}\left\{\begin{array}{l}
\frac{\partial w_{s}}{\partial y} \\
\frac{\partial x}{\partial x}
\end{array}\right\}
\end{aligned}
$$

The strain and stress fields in a medium are linked by constitutive laws; these laws characterize the mechanical behavior of the medium. Consequently, the linear elastic relationship for an FGM plate can be written in the following matrix form: 

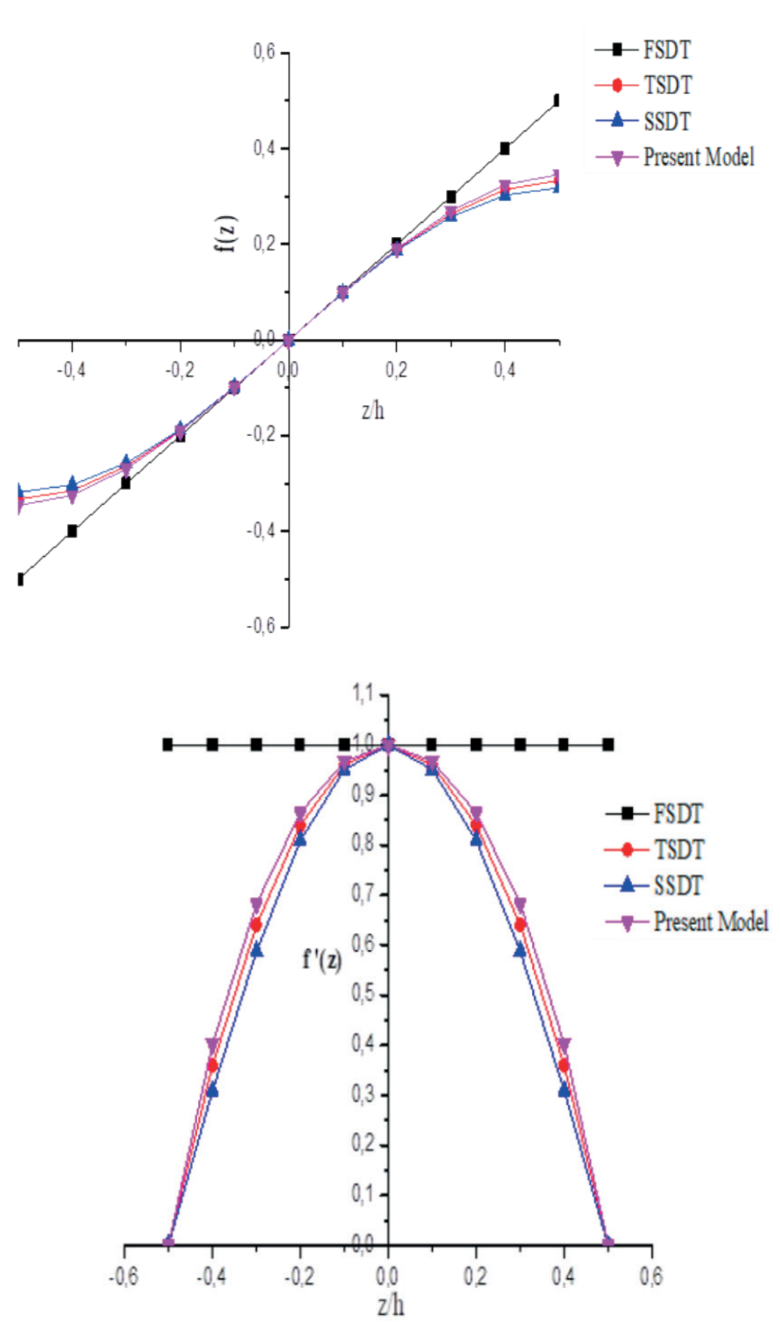

Fig. 2 Convergence of the proposed functionand its derivative with those of (FSDT), (TSDT), (SSDT) and present models

$$
\left\{\begin{array}{l}
\sigma_{x x} \\
\sigma_{y y} \\
\tau_{x y} \\
\tau_{y z} \\
\tau_{x z}
\end{array}\right\}=\left[\begin{array}{ccccc}
C_{11}(z) & C_{12}(z) & 0 & 0 & C_{16}(z) \\
C_{12}(z) & C_{22}(z) & 0 & 0 & C_{26}(z) \\
0 & 0 & C_{44}(z) & C_{45}(z) & 0 \\
0 & 0 & C_{45}(z) & C_{55}(z) & 0 \\
C_{16}(z) & C_{26}(z) & 0 & 0 & C_{66}(z)
\end{array}\right]\left\{\begin{array}{l}
\varepsilon_{x x} \\
\varepsilon_{y y} \\
\gamma_{x y} \\
\gamma_{y z} \\
\gamma_{x z}
\end{array}\right\} .
$$

The elasticity relationships are generally expressed as a function of the rigidity constants which themselves are expressed in terms of the elasticity modulus that are determined by mechanical tests in which the material used is subjected to a particular stress and deformation state. The terms $C_{i j}(z)$ represent the stiffness constants which depend on the constituents of the FGM material.

$$
\begin{aligned}
& C_{11}(z)=C_{22}(z)=\frac{E(z)}{1-v^{2}}, C_{12}(z)=\frac{v E(z)}{1-v^{2}} \\
& C_{44}(z)=C_{55}(z)=C_{66}(z)=\frac{E(z)}{2(1+v)} \\
& C_{16}(z)=C_{26}(z)=C_{45}(z)=0
\end{aligned}
$$

Furthermore, the parameters $E(z)$ and $v$ are the Young's modulus and Poisson's ratio of the material; they depend on the characteristics of the FGM plate. The mechanical properties of the FGM plate containing ceramic and metal, which are uniformly distributed, are given by the power law hypothesis which is written as the general mixing rule under the following form:

$P(z)=\left(P_{c}-P_{m}\right)\left(\frac{z}{h}+\frac{1}{2}\right)^{p}+P_{m}$.

where: $P$ denotes the effective material property, and the subscripts $c$ and $m$ stand for ceramic and metal, respectively.

In addition, the equations expressing Young's modulus $E(z)$ and density $\rho(z)$ of the material of a functionally graded plate can be written as follows:

$$
\begin{aligned}
& E(z)=\left(E_{c}-E_{m}\right)\left(\frac{z}{h}+\frac{1}{2}\right)^{p}+E_{m}, \\
& \rho(z)=\left(\rho_{c}-\rho_{m}\right)\left(\frac{z}{h}+\frac{1}{2}\right)^{p}+\rho_{m},
\end{aligned}
$$

where: $E_{c}, E_{m}, \rho_{c}$ and $\rho_{m}$ are Young's modulus and volume densities corresponding to ceramic and metal, respectively; $p$ represents the exponent of the volume fraction which takes only values greater or equal to zero $(0 \leq p \leq \infty)$. The value zero corresponds to a ceramic plate.

In our study, the Poisson's ratio is assumed to be constant.

\section{Non-local viscoelastic theory}

\subsection{Nonlocal elasticity theory}

Given the importance of the intermolecular attractions of the material, the theory of nonlocal effect developed by Eringen suggests that the stresses at a reference point $x$ in the body depend not only on the deformations at $x$ but also on the deformations at all points of the body (scale effect). The constitutive relation of the elastic constitutive law of a nanosolid is expressed by the following relation:

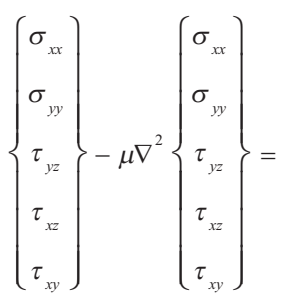

$$
\left[\begin{array}{ccccc}
C_{11}(z) & C_{12}(z) & 0 & 0 & C_{16}(z) \\
C_{12}(z) & C_{22}(z) & 0 & 0 & C_{26}(z) \\
0 & 0 & C_{44}(z) & C_{45}(z) & 0 \\
0 & 0 & C_{45}(z) & C_{55}(z) & 0 \\
C_{16}(z) & C_{26}(z) & 0 & 0 & C_{66}(z)
\end{array}\right]\left\{\begin{array}{l}
\varepsilon_{x x} \\
\varepsilon_{y y} \\
\gamma_{y z} \\
\gamma_{x z} \\
\gamma_{x y}
\end{array}\right\}
$$


The term $\nabla^{2}$ is the Laplace operator in two-dimensional Cartesian coordinates. It is expressed by:

$\nabla^{2}=\frac{\partial^{2}}{\partial x^{2}}+\frac{\partial^{2}}{\partial y^{2}}$.

The parameter $\mu=\left(e_{0} a\right)^{2}$ is the nonlocal parameter which depends on the material constant $e_{0}$ and the internal characteristic $a$ (lattice parameter, crack length or molecular diameters).

\subsection{Theory of viscoelasticity}

The theory of viscoelasticity can take into account materials capable of storing and dissipating mechanical energy. On the basis of the Kelvin-Voigt model on elastic materials with viscoelastic structural damping coefficient $\eta$, the rigidities $C_{i j}$ which depend on Young's modulus $E$ and shear modulus $G$ are replaced by the operators, $C_{i j}\left(1+\eta \frac{\partial}{\partial t}\right)$. Therefore, Eq. (6) can be written again as follows:

$$
\left\{\begin{array}{l}
\sigma_{x x} \\
\sigma_{y y} \\
\tau_{y z} \\
\tau_{x z} \\
\tau_{x y}
\end{array}\right\}=\left(1+\eta \frac{\partial}{\partial t}\right)\left[\begin{array}{ccccc}
C_{11}(z) & C_{12}(z) & 0 & 0 & C_{16}(z) \\
C_{12}(z) & C_{22}(z) & 0 & 0 & C_{26}(z) \\
0 & 0 & C_{44}(z) & C_{45}(z) & 0 \\
0 & 0 & C_{45}(z) & C_{55}(z) & 0 \\
C_{16}(z) & C_{26}(z) & 0 & 0 & C_{66}(z)
\end{array}\right]\left\{\begin{array}{l}
\varepsilon_{x x} \\
\varepsilon_{y y} \\
\gamma_{y z} \\
\gamma_{x z} \\
\gamma_{x y}
\end{array}\right\}
$$

The theory of the nonlocal viscoelasticity principle, previously developed by several researchers, initially assumed a combination of the models of nonlocal elasticity and viscoelasticity. Therefore, for nonlocal viscoelastic plates, the nonlocal viscoelastic stress field can be expressed in the following form:

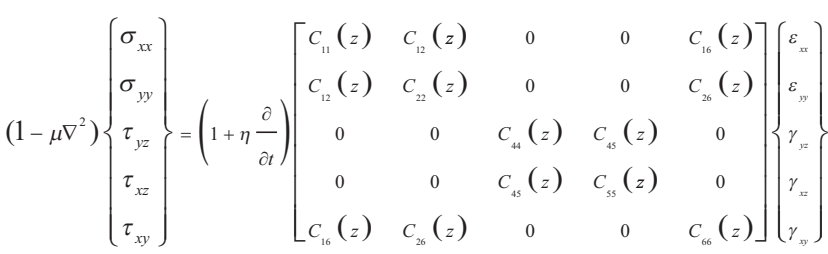

\section{Equilibrium equations}

To establish the equations governing the equilibrium of an FGM viscoelastic nanoplate resting on the WinklerPasternak viscoelastic foundation, the principle of virtual work (Hamilton's principle) can be used in the following form:

$$
\int_{t_{0}}^{t_{1}} \Pi d t=\int_{t_{0}}^{t_{1}}\left(\delta U-\delta K-\delta W_{f e}\right) d t=0 .
$$

In this case, the virtual strain energy may be expressed as:

$$
\delta U=\iiint_{V}\left(\sigma_{x x} \delta \varepsilon_{x x}+\sigma_{y y} \delta \varepsilon_{y y}+\tau_{x y} \delta \gamma_{x y}+\tau_{x z} \delta \gamma_{x z}+\tau_{y z} \delta \gamma_{y z}\right) d V .
$$

By substituting Eq. (5) into Eq. (13), the virtual strain energy becomes:

$$
\begin{aligned}
& \delta U=\iint_{A}\left[\begin{array}{l}
N_{x x} \delta\left(\frac{\partial u_{0}}{\partial x}\right)-M_{x x} \delta\left(\frac{\partial^{2} w_{b}}{\partial x^{2}}\right)+S_{x x} \delta\left(\frac{\partial w_{s}}{\partial x^{2}}\right) \\
+N_{y y} \delta\left(\frac{\partial v_{0}}{\partial y}\right)-M_{y y} \delta\left(\frac{\partial^{2} w_{b}}{\partial y^{2}}\right)+S_{y y} \delta\left(\frac{\partial w_{s}}{\partial y^{2}}\right)+
\end{array}\right. \\
& \left.N_{x x} \delta\left(\frac{\partial u_{0}}{\partial y}+\frac{\partial v_{0}}{\partial x}\right)-2 M_{x y} \delta\left(\frac{\partial^{2} w_{b}}{\partial x \partial y}\right)+S_{x y} \delta\left(\frac{\partial^{2} w_{s}}{\partial x \partial y}\right)\right]_{d A,} \\
& +Q_{x z} \delta\left(\frac{\partial w_{s}}{\partial x}\right)+Q_{y z} \delta\left(\frac{\partial w_{s}}{\partial y}\right)
\end{aligned}
$$

where: $N_{i j}, M_{i j}$ and $Q_{i j}$ are the normal forces, bending moments and shear forces, respectively. The unusual term $S_{i j}$ has the dimension of a moment that is induced by $(f(z)-z)$ in the displacement field; it is defined as:

$$
\left(N_{i j}, M_{i j}, S_{i j}, Q_{i j}\right)=\int_{-\frac{h}{2}}^{\frac{h}{2}} \sigma_{i j}\left(1, z, f(z)-z, \frac{\partial f(z)}{\partial z}\right) d z .
$$

In addition, the expressions of local elastic forces and moments are given by:

$$
\begin{aligned}
& \left\{\begin{array}{l}
N_{x x} \\
N_{y y} \\
N_{x y} \\
M_{x x} \\
M_{y y} \\
M_{x y} \\
S_{x x} \\
S_{y y} \\
S_{x y}
\end{array}\right\}=\left[\begin{array}{lllllllll}
A_{11} & A_{12} & A_{16} & B_{11} & B_{12} & B_{16} & B_{11}^{f} & B_{12}^{f} & B_{16}^{f} \\
A_{12} & A_{22} & A_{26} & B_{12} & B_{22} & B_{26} & B_{12}^{f} & B_{22}^{f} & B_{26}^{f} \\
A_{16} & A_{26} & A_{66} & B_{16} & B_{26} & B_{66} & B_{16}^{f} & B_{26}^{f} & B_{66}^{f} \\
B_{11} & B_{12} & B_{16} & D_{11} & D_{12} & D_{16} & D_{11}^{f} & D_{12}^{f} & D_{16}^{f} \\
B_{12} & B_{22} & B_{26} & D_{12} & D_{22} & D_{26} & D_{12}^{f} & D_{22}^{f} & D_{26}^{f} \\
B_{16} & B_{26} & B_{66} & D_{16} & D_{26} & D_{66} & D_{16}^{f} & D_{26}^{f} & D_{66}^{f} \\
B_{11}^{f} & B_{12}^{f} & B_{16}^{f} & D_{11}^{f} & D_{12}^{f} & D_{16}^{f} & F_{11}^{f} & F_{12}^{f} & F_{16}^{f} \\
B_{12}^{f} & B_{22}^{f} & B_{26}^{f} & D_{12}^{f} & D_{22}^{f} & D_{26}^{f} & F_{12}^{f} & F_{22}^{f} & F_{26}^{f} \\
B_{16}^{f} & B_{26}^{f} & B_{66}^{f} & D_{16}^{f} & D_{26}^{f} & D_{66}^{f} & F_{16}^{f} & F_{26}^{f} & F_{66}^{f}
\end{array}\right]\left\{\begin{array}{c}
u_{0, x} \\
v_{0, y} \\
u_{0, y}+v_{0, x} \\
-w_{s, x x} \\
-w_{b, y y} \\
-2 w_{b, y y} \\
w_{s, x} \\
w_{s, y} \\
2 w_{s, x y}
\end{array}\right\} \\
& \left\{\begin{array}{l}
Q_{x z} \\
Q_{y z}
\end{array}\right\}=\left[\begin{array}{cc}
A_{44}^{f} & A_{45}^{f} \\
A_{45}^{f} & A_{55}^{f}
\end{array}\right]\left\{\begin{array}{l}
w_{s, x} \\
w_{s, y}
\end{array}\right\} .
\end{aligned}
$$

Similarly, the elements of the matrix given by Eqs. (17) and (18) can be expressed as:

$$
\begin{aligned}
& \left\{A_{i j}, B_{i j}, D_{i j}, B_{i j}^{f}\right\}=\int C_{i j}(z)\left\{1, z, z^{2}, f(z)-z\right\} d z \quad i, j=1,2,6, \\
& \left\{D_{i j}^{f}, F_{i j}^{f}\right\}=\int C_{i j}(z)\left\{z(f(z)-z),(f(z)-z)^{2}\right\} d z \quad i, j=1,2,6, \\
& \left\{A_{i j}^{f}\right\}=\int C_{i j}(z)\left\{\left(\frac{\partial f(z)}{\partial z}\right)^{2}\right\} d z \quad i, j=4,5 .
\end{aligned}
$$

On the other hand, the kinetic energy is defined as follows: 


$$
K=\frac{1}{2} \iiint_{V}\left[\left(\frac{\partial U}{\partial t}\right)^{2}+\left(\frac{\partial V}{\partial t}\right)^{2}+\left(\frac{\partial W}{\partial t}\right)^{2}\right] d V
$$

When Eq. (1) is substituted into Eq. (20), the virtual kinetic energy becomes:

$$
\begin{aligned}
& \delta K=\iint_{A}\left[\begin{array}{l}
\left(I_{1} \frac{\partial u_{0}}{\partial t}-I_{2} \frac{\partial^{2} w_{b}}{\partial x \partial t}+I_{4} \frac{\partial w_{s}}{\partial x \partial t}\right) \delta\left(\frac{\partial u_{0}}{\partial t}\right) \\
-\left(I_{2} \frac{\partial u_{0}}{\partial t}-\frac{\partial^{2} w_{0}}{\partial x \partial t}+I_{5} \frac{\partial w_{s}}{\partial x \partial t}\right) \delta\left(\frac{\partial^{2} w_{b}}{\partial x \partial t}\right)+
\end{array}\right. \\
& \left(I_{4} \frac{\partial u_{0}}{\partial t}-I_{5} \frac{\partial^{2} w_{b}}{\partial x \partial t}+I_{6} \frac{\partial^{2} w_{s}}{\partial x \partial t}\right) \delta\left(\frac{\partial^{2} w_{s}}{\partial x \partial t}\right)+ \\
& \left(I_{1} \frac{\partial v_{0}}{\partial t}-I_{2} \frac{\partial^{2} w_{b}}{\partial y \partial t}+I_{4} \frac{\partial^{2} w_{s}}{\partial y \partial t}\right) \delta\left(\frac{\partial v_{0}}{\partial t}\right)- \\
& \left(I_{2} \frac{\partial v_{0}}{\partial t}-\frac{\partial^{2} w_{b}}{\partial y \partial t}+I_{5} \frac{\partial^{2} w_{s}}{\partial y \partial t}\right) \delta\left(\frac{\partial^{2} w_{b}}{\partial y \partial t}\right)+ \\
& \left(I_{4} \frac{\partial v_{0}}{\partial t}-I_{5} \frac{\partial^{2} w_{b}}{\partial v \partial t}+I_{6} \frac{\partial^{2} w_{s}}{\partial y \partial t}\right) \delta\left(\frac{\partial^{2} w_{s}}{\partial y \partial t}\right)+ \\
& \left.I_{1}\left(\frac{\partial w_{b}}{\partial t}+\frac{\partial w_{s}}{\partial t}\right) \delta\left(\frac{\partial w_{b}}{\partial t}+\frac{\partial w_{s}}{\partial t}\right)\right] d V .
\end{aligned}
$$

Where: $I_{1}, I_{2}, I_{3}, I_{4}, I_{5}$ and $I_{6}$ are the mass inertias which are defined by:

$$
\left\{I_{i}\right\}=\int_{-\frac{h}{2}}^{\frac{h}{2}} \rho(z)\left\{1, z, z^{2}, f(z)-z, z(f(z)-z),(f(z)-z)^{2}\right\} d z, i=1 . .6 .
$$

The virtual work done by the viscoelastic WinklerPasternak foundation is given by:

$$
\begin{aligned}
& \delta W_{f e}=\iint_{A} f_{e}(x, y) \delta W d A \\
& \delta W_{f e}=\iint_{A}\left(k_{w} W-k_{p}\left(\frac{\partial^{2}}{\partial x^{2}}+\frac{\partial^{2}}{\partial y^{2}}\right) W+c_{d} \frac{\partial W}{\partial t}\right) \delta W d A,
\end{aligned}
$$

where: $k_{w}, k_{p}$ and $c_{d}$ are, respectively, the Winkler coefficient, Pasternak coefficient and damping parameter.

By performing integration by parts of Eq. (15), Eq. (21) and Eq. (23), one obtains the equations of motion used in the refined FGM plate theory. These equations may be applied to homogeneous thin or thick laminated plates. They take into account the transverse shearing effect. Therefore:

$$
\begin{aligned}
& \delta u_{0}: \frac{\partial N_{x x}}{\partial x}+\frac{\partial N_{x y}}{\partial y}=I_{1} \frac{\partial^{2} u_{0}}{\partial t^{2}}-I_{2} \frac{\partial^{3} w_{b}}{\partial x \partial t^{2}}+I_{4} \frac{\partial^{3} w_{s}}{\partial x \partial t^{2}}, \\
& \delta v_{0}: \frac{\partial N_{x y}}{\partial x}+\frac{\partial N_{y y}}{\partial y}=I_{1} \frac{\partial^{2} v_{0}}{\partial t^{2}}-I_{2} \frac{\partial^{3} w_{b}}{\partial y \partial t^{2}}+I_{4} \frac{\partial^{3} w_{s}}{\partial y \partial t^{2}}, \\
& \delta w_{b}: \frac{\partial^{2} M_{x x}}{\partial x^{2}}+2 \frac{\partial^{2} M_{x y}}{\partial x \partial y}+\frac{\partial^{2} M_{y y}}{\partial y^{2}}=f_{e}+I_{1}\left(\frac{\partial^{2} w_{b}}{\partial t^{2}}+\frac{\partial^{2} w_{s}}{\partial t^{2}}\right) \\
& +I_{2}\left(\frac{\partial^{3} u_{0}}{\partial x \partial t^{2}}+\frac{\partial^{3} v_{0}}{\partial y \partial t^{2}}\right)-I_{3}\left(\frac{\partial^{4} w_{b}}{\partial x^{2} \partial t^{2}}+\frac{\partial^{4} w_{b}}{\partial y^{2} \partial t^{2}}\right)+ \\
& I_{5}\left(\frac{\partial^{4} w_{s}}{\partial x^{2} \partial t^{2}}+\frac{\partial^{4} w_{s}}{\partial y^{2} \partial t^{2}}\right), \\
& \delta w_{s}: \frac{\partial^{2} S_{x x}}{\partial x^{2}}+2 \frac{\partial^{2} S_{x y}}{\partial x \partial y}+\frac{\partial^{2} S_{y y}}{\partial y^{2}}-\frac{\partial Q_{x z}}{\partial x}-\frac{\partial Q_{y z}}{\partial y}=f_{e}+ \\
& I_{1}\left(\frac{\partial^{2} w_{b}}{\partial t^{2}}+\frac{\partial^{2} w_{s}}{\partial t^{2}}\right)+I_{4}\left(\frac{\partial^{3} u_{0}}{\partial x \partial t^{2}}+\frac{\partial^{3} v_{0}}{\partial y \partial t^{2}}\right)- \\
& I_{5}\left(\frac{\partial^{4} w_{b}}{\partial x^{2} \partial t^{2}}+\frac{\partial^{4} w_{b}}{\partial y^{2} \partial t^{2}}\right)+I_{6}\left(\frac{\partial^{4} w_{s}}{\partial x^{2} \partial t^{2}}+\frac{\partial^{4} w_{s}}{\partial y^{2} \partial t^{2}}\right) .
\end{aligned}
$$


The internal forces with viscoelastic and nonlocal parameters can be expressed in the following form:

$$
\left\{\begin{array}{l}
Q_{x z} \\
Q_{y z}
\end{array}\right\}-\mu \nabla^{2}\left\{\begin{array}{l}
Q_{x z} \\
Q_{y z}
\end{array}\right\}=\left(1+\eta \frac{\partial}{\partial t}\right)\left[\begin{array}{ll}
A_{44}^{f} & A_{45}^{f} \\
A_{45}^{f} & A_{55}^{f}
\end{array}\right]\left\{\begin{array}{l}
w_{s, x} \\
w_{s, y}
\end{array}\right\} .
$$

The equations governing FGM viscoelastic nanoplates resting on a viscoelastic medium having three parameters in the case of free vibrations, in terms of displacements, are obtained by introducing the constitutive Eq. (25) and Eq. (26) in the equations of equilibrium (24). This makes it possible to obtain the four fundamental relations of the refined theory of nonlocal viscoelastic plates of FGM materials:

$$
\begin{aligned}
& \delta u_{0}: \mu \frac{\partial^{2}}{\partial x^{2}}\left(I_{1} \frac{\partial^{2} u_{0}}{\partial t^{2}}-I_{2} \frac{\partial^{3} w_{b}}{\partial x \partial t^{2}}+I_{4} \frac{\partial^{3} w_{s}}{\partial x \partial t^{2}}\right) \\
& +\mu \frac{\partial^{2}}{\partial y^{2}}\left(I_{1} \frac{\partial^{2} u_{0}}{\partial t^{2}}-I_{2} \frac{\partial^{3} w_{b}}{\partial x \partial t^{2}}+I_{4} \frac{\partial^{3} w_{s}}{\partial x \partial t^{2}}\right)+ \\
& \left(1+\frac{\partial}{\partial t}\right)\left(\begin{array}{c}
A_{11} \frac{\partial^{2} u_{0}}{\partial x^{2}}-B_{11} \frac{\partial^{3} w_{b}}{\partial x^{3}}+B_{11}^{f} \frac{\partial^{3} w_{s}}{\partial x^{3}}+ \\
A_{12} \frac{\partial^{2} v_{0}}{\partial x \partial y}-B_{12} \frac{\partial^{3} w_{b}}{\partial y^{2} \partial x}+B_{12}^{f} \frac{\partial^{3} w_{s}}{\partial y^{2} \partial x}
\end{array}\right)+ \\
& \left(1+\frac{\partial}{\partial t}\right)\left(\begin{array}{l}
A_{66}\left(\frac{\partial^{2} u_{0}}{\partial y^{2}}+\frac{\partial^{2} v_{0}}{\partial x \partial y}\right)+2 B_{66} \frac{\partial^{3} w_{b}}{\partial x \partial y^{2}}+ \\
2 B_{66}^{f} \frac{\partial^{3} w_{s}}{\partial x \partial y^{2}}
\end{array}\right) \\
& =I_{1} \frac{\partial^{2} u_{0}}{\partial t^{2}}-I_{2} \frac{\partial^{3} w_{b}}{\partial x \partial t^{2}}+I_{4} \frac{\partial^{3} w_{s}}{\partial x \partial t^{2}} \\
& \delta v_{0}: \mu \frac{\partial^{2}}{\partial x^{2}}\left(I_{1} \frac{\partial^{2} v_{0}}{\partial t^{2}}-I_{2} \frac{\partial^{3} w_{b}}{\partial y \partial t^{2}}+I_{4} \frac{\partial^{3} w_{s}}{\partial y \partial t^{2}}\right) \\
& +\mu \frac{\partial^{2}}{\partial y^{2}}\left(I_{1} \frac{\partial^{2} v_{0}}{\partial t^{2}}-I_{2} \frac{\partial^{3} w_{b}}{\partial y \partial t^{2}}+I_{4} \frac{\partial^{3} w_{s}}{\partial y \partial t^{2}}\right)+ \\
& \left(1+\frac{\partial}{\partial t}\right)\left(\begin{array}{l}
A_{12} \frac{\partial^{2} u_{0}}{\partial x \partial y}-B_{12} \frac{\partial^{3} w_{b}}{\partial x^{2} \partial y}+B_{12}^{f} \frac{\partial^{3} w_{s}}{\partial x^{2} \partial y} \\
+A_{22} \frac{\partial^{2} v_{0}}{\partial y^{2}}-B_{22} \frac{\partial^{3} w_{b}}{\partial y^{3}}+B_{22}^{f} \frac{\partial^{3} w_{s}}{\partial y^{3}}
\end{array}\right)+ \\
& \left(1+\frac{\partial}{\partial t}\right)\left(\begin{array}{l}
A_{66}\left(\frac{\partial^{2} u_{0}}{\partial x \partial y}+\frac{\partial^{2} v_{0}}{\partial x^{2}}\right)-2 B_{66} \frac{\partial^{3} w_{b}}{\partial x^{2} \partial y}+ \\
2 B_{66}^{f} \frac{\partial^{3} w_{s}}{\partial x^{2} \partial y}
\end{array}\right) \\
& =I_{1} \frac{\partial^{2} v_{0}}{\partial t^{2}}-I_{2} \frac{\partial^{3} w_{b}}{\partial y \partial t^{2}}+I_{4} \frac{\partial^{3} w_{s}}{\partial y \partial t^{2}}
\end{aligned}
$$

$+\left(1+\frac{\partial}{\partial t}\right)\left(\begin{array}{l}B_{12}^{f} \frac{\partial^{3} u_{0}}{\partial y^{2} \partial x}-D_{12}^{f} \frac{\partial^{4} w_{b}}{\partial x^{2} \partial y^{2}}+F_{12}^{f} \frac{\partial^{4} w_{s}}{\partial x^{2} \partial y^{2}}+A_{55}^{f} \frac{\partial^{2} w_{s}}{\partial x^{2}} \\ +B_{22}^{f} \frac{\partial^{3} v_{0}}{\partial y^{3}}-D_{22}^{f} \frac{\partial^{4} w_{b}}{\partial y^{4}}+F_{22}^{f} \frac{\partial^{4} w_{s}}{\partial y^{4}}+A_{55}^{f} \frac{\partial^{2} w_{s}}{\partial y^{2}}\end{array}\right)+$

$2\left(1+\frac{\partial}{\partial t}\right)\left(B_{66}^{f}\left(\frac{\partial^{3} u_{0}}{\partial x \partial y^{2}}+\frac{\partial^{3} v_{0}}{\partial x^{2} \partial y}\right)-2 D_{66}^{f} \frac{\partial^{3} w_{b}}{\partial x^{2} \partial y^{2}}+2 F_{66}^{f} \frac{\partial^{3} w_{s}}{\partial x^{2} \partial y^{2}}\right)$

$=f_{e}+I_{1}\left(\frac{\partial^{2} w_{b}}{\partial t^{2}}+\frac{\partial^{2} w_{s}}{\partial t^{2}}\right)+I_{4}\left(\frac{\partial^{3} u_{0}}{\partial x \partial t^{2}}+\frac{\partial^{3} v_{0}}{\partial y \partial t^{2}}\right)$

$-I_{5}\left(\frac{\partial^{4} w_{b}}{\partial x^{2} \partial t^{2}}+\frac{\partial^{4} w_{b}}{\partial y^{2} \partial t^{2}}\right)+I_{6}\left(\frac{\partial^{4} w_{s}}{\partial x^{2} \partial t^{2}}+\frac{\partial^{4} w_{s}}{\partial y^{2} \partial t^{2}}\right)$ 


\section{Analytical solution}

In general, rectangular plates are classified according to the type of support used. The goal is to find the analytical solutions of Eqs. (27) to (30) under various boundary conditions imposed on the lateral edges, as shown in Table 2. The edges of the plate are either embedded or simply supported. The boundary conditions used are:

Simply supported plate $(S)$ along direction $\mathrm{x}$ :

$$
\begin{aligned}
& \text { at } \quad x=0, a: \\
& \bullet v_{0}=w_{b}=\frac{\partial w_{s}}{\partial y}=0 \\
& \bullet N_{x x}=A_{11} \frac{\partial u_{0}}{\partial x}+A_{16}\left(\frac{\partial u_{0}}{\partial y}+\frac{\partial v_{0}}{\partial x}\right)-B_{11} \frac{\partial^{2} w_{s}}{\partial x^{2}}+B_{11}^{f} \frac{\partial w_{s}}{\partial x} \\
& +A_{12} \frac{\partial v_{0}}{\partial y}-B_{12} \frac{\partial^{2} w_{b}}{\partial y^{2}}-2 B_{16} \frac{\partial^{2} w_{b}}{\partial y \partial x}+B_{12}^{f} \frac{\partial w_{s}}{\partial y}+2 B_{16}^{f} \frac{\partial w_{s}}{\partial y \partial x}=0 \\
& \bullet M_{x x}=B_{11} \frac{\partial u_{0}}{\partial x}+B_{16}\left(\frac{\partial u_{0}}{\partial y}+\frac{\partial v_{0}}{\partial x}\right)-D_{11} \frac{\partial^{2} w_{s}}{\partial x^{2}}+D_{11}^{f} \frac{\partial w_{s}}{\partial x}+ \\
& B_{12} \frac{\partial v_{0}}{\partial y}-D_{12} \frac{\partial^{2} w_{b}}{\partial y^{2}}-2 D_{16} \frac{\partial^{2} w_{b}}{\partial y \partial x}+D_{12}^{f} \frac{\partial w_{s}}{\partial y}+2 D_{16}^{f} \frac{\partial w_{s}}{\partial y \partial x}=0 \\
& \bullet S_{x x}=B_{11}^{f} \frac{\partial u_{0}}{\partial x}+B_{16}^{f}\left(\frac{\partial u_{0}}{\partial y}+\frac{\partial v_{0}}{\partial x}\right)-D_{11}^{f} \frac{\partial^{2} w_{s}}{\partial x^{2}}+F_{11}^{f} \frac{\partial w_{s}}{\partial x}+ \\
& B_{12}^{f} \frac{\partial v_{0}}{\partial y}-D_{12}^{f} \frac{\partial^{2} w_{b}}{\partial y^{2}}-2 D_{16}^{f} \frac{\partial^{2} w_{b}}{\partial y \partial x}+F_{12}^{f} \frac{\partial w_{s}}{\partial y}+2 F_{16}^{f} \frac{\partial w_{s}}{\partial y \partial x}=0
\end{aligned}
$$

Simply supported plate $(S)$ along direction y:

$$
\begin{aligned}
& \text { at } \quad y=0, b: \\
& \bullet u_{0}=w_{b}=\frac{\partial w_{s}}{\partial x}=0 \\
& \bullet N_{y y}=A_{12} \frac{\partial u_{0}}{\partial x}+A_{26}\left(\frac{\partial u_{0}}{\partial y}+\frac{\partial v_{0}}{\partial x}\right)-B_{12} \frac{\partial^{2} w_{s}}{\partial x^{2}}+B_{12}^{f} \frac{\partial w_{s}}{\partial x} \\
& +A_{22} \frac{\partial v_{0}}{\partial y}-B_{22} \frac{\partial^{2} w_{b}}{\partial y^{2}}-2 B_{26} \frac{\partial^{2} w_{b}}{\partial y \partial x}+B_{22}^{f} \frac{\partial w_{s}}{\partial y}+2 B_{26}^{f} \frac{\partial w_{s}}{\partial y \partial x}=0 \\
& \bullet M_{y y}=B_{12} \frac{\partial u_{0}}{\partial x}+B_{26}\left(\frac{\partial u_{0}}{\partial y}+\frac{\partial v_{0}}{\partial x}\right)-D_{12} \frac{\partial^{2} w_{s}}{\partial x^{2}}+D_{12}^{f} \frac{\partial w_{s}}{\partial x}+ \\
& B_{22} \frac{\partial v_{0}}{\partial y}-D_{22} \frac{\partial^{2} w_{b}}{\partial y^{2}}-2 D_{26} \frac{\partial^{2} w_{b}}{\partial y \partial x}+D_{22}^{f} \frac{\partial w_{s}}{\partial y}+2 D_{26}^{f} \frac{\partial w_{s}}{\partial y \partial x}=0 \\
& \bullet S_{y y}=B_{12}^{f} \frac{\partial u_{0}}{\partial x}+B_{26}^{f}\left(\frac{\partial u_{0}}{\partial y}+\frac{\partial v_{0}}{\partial x}\right)-D_{12}^{f} \frac{\partial^{2} w_{s}}{\partial x^{2}}+F_{12}^{f} \frac{\partial w_{s}}{\partial x}+ \\
& B_{22}^{f} \frac{\partial v_{0}}{\partial y}-D_{22}^{f} \frac{\partial^{2} w_{b}}{\partial y^{2}}-2 D_{26}^{f} \frac{\partial^{2} w_{b}}{\partial y \partial x}+F_{22}^{f} \frac{\partial w_{s}}{\partial y}+2 F_{26}^{f} \frac{\partial w_{s}}{\partial y \partial x}=0
\end{aligned}
$$

Embedded plate (C)

$$
\text { at } \quad x=0, a, y=0, b \quad, u_{0}=v_{0}=w_{b}=\frac{\partial w_{s}}{\partial x}=\frac{\partial w_{s}}{\partial y}=0
$$

Table 2 The admissible functions $X_{i}(x)$ and $Y_{i}(y)$ as given by Kiasat et al. [32] and Sobhy [38]

\begin{tabular}{lcc}
\hline $\mathrm{BC}$ & $X_{i}(x)$ & $Y_{i}(y)$ \\
\hline $\mathrm{SSSS}$ & $\sin (\alpha x)$ & $\sin (\beta y)$ \\
$\mathrm{CCCC}$ & $1-\cos (2 \alpha x)$ & $1-\cos (2 \beta y)$ \\
$\mathrm{CCSS}$ & $1-\cos (2 \alpha x)$ & $\sin (\beta y)$ \\
$\mathrm{CSCS}$ & $\cos \left(\frac{3 \alpha x}{2}\right)-\cos \left(\frac{\alpha x}{2}\right)$ & $\cos \left(\frac{3}{2} \beta y\right)-\cos \left(\frac{1}{2} \beta y\right)$ \\
$\mathrm{CSSS}$ & $\cos \left(\frac{3}{2} \alpha x\right)-\cos \left(\frac{1}{2} \alpha x\right)$ & $\sin (\beta y)$ \\
$\mathrm{CCSC}$ & $1-\cos (2 \alpha x)$ & $\cos \left(\frac{3}{2} \beta y\right)-\cos \left(\frac{1}{2} \beta y\right)$ \\
\hline
\end{tabular}

The Navier method is used under the specified boundary conditions to find the analytical solution to Eqs. (27)-(29) and (30). The displacement functions, which satisfy the boundary conditions, are expressed as the following Fourier series:

$$
\left\{\begin{array}{c}
u_{0} \\
v_{0} \\
w_{b} \\
w_{s}
\end{array}\right\}=\sum_{i=1}^{\infty} \sum_{j=1}^{\infty}\left\{\begin{array}{l}
U_{i j} \frac{\partial X_{i}(x)}{\partial x} Y_{j}(y) \\
V_{i j} X_{i}(x) \frac{\partial Y_{j}(y)}{\partial y} \\
W_{b i j} X_{i}(x) Y_{j}(y) \\
W_{s i j} X_{i}(x) Y_{j}(y)
\end{array}\right\} e^{\lambda t} .
$$

The quantities $U_{i j}, V_{i j}, W_{b i j}$ and $W_{s i j}$ refer to the amplitudes, and $\lambda$ corresponds to the complex eigen-frequency of the (ith, $j$ th) vibrational eigen mode, with $\alpha=i \pi / a$, $\beta=i \pi / b$

Using Eq. (34) in the equations of motion Eqs. (27) to (30) gives, for any fixed values of $i$ and $j$, in the case of free vibrations, the eigenvalue equations can expressed as:

$\left[\begin{array}{llll}L_{11} & L_{12} & L_{13} & L_{14} \\ L_{21} & L_{22} & L_{23} & L_{24} \\ L_{31} & L_{32} & L_{33} & L_{34} \\ L_{41} & L_{42} & L_{43} & L_{44}\end{array}\right]\left\{\begin{array}{c}U_{i j} \\ V_{i j} \\ W_{b i j} \\ W_{s i j}\end{array}\right\}=\{0\}$.

It is worth noting that $\left[U_{i j}, V_{i j}, W_{b i j}, W_{s i j}\right]$ is the displacement, and $[L]$ is the global matrix of the system. The elements $L_{i j}=L_{j i}$ of the coefficients matrix are given in the Appendix. For non-trivial solutions of Eqs. (27) to (30), the following determinants should be set equal to zero to find the eigenfrequency $\lambda$ : 


$$
\operatorname{Det}([L])=0
$$

Note also that the frequency and parameters used in this study are dimensionless and can be written in the following form:

$$
\begin{aligned}
& K_{w}=\frac{k_{w} a^{4}}{D_{c}}, K_{p}=\frac{k_{p} a^{2}}{D_{c}}, \psi=\frac{\eta}{a^{2}} \sqrt{\frac{D_{c}}{\rho_{c} h}}, \\
& C_{d}=\frac{c_{d} a^{2}}{\sqrt{\rho_{c} h D_{c}}}, D_{c}=\frac{E_{c} h^{3}}{12\left(1-v^{2}\right)} .
\end{aligned}
$$

Finally, the dimensionless eigenfrequency may be expressed as follows:

$$
\varpi=-\Omega\left(\xi \pm i \sqrt{1-\xi^{2}}\right), \Omega=\lambda a^{2} \sqrt{\frac{\rho_{c} h}{D_{C}}}
$$

Here $\Omega$ and $\xi$ are respectively the dimensionless undamped frequency and damping ratio.

Regarding the expression under the square root $\xi \sqrt{1-\xi^{2}}$, three different cases may be considered:

$0<\xi<1$, in the case of underdamped vibration of nanoplate,

$\xi=1$, in the case of critically damped vibration of nanoplate,

$\xi>1$, in the case of overdamped vibration of nanoplate.

\section{Comparative studies}

In order to validate the suggested model, the authors decided to compare the results obtained with those previously found by several other researchers. The first example involves three natural frequencies, given by the present model for various values of the nonlocal parameter $(\mu)$, which are compared with those given by the nonlocal higher-order theory for simply supported plates using Reddy's third-order shear deformation theory (TSDT) [25], as presented in Table 3.

The approach followed here is pretty straightforward. Indeed, it was revealed that for the first vibrational mode, $\left(\Omega_{11}\right)$ second vibrational mode $\left(\Omega_{22}\right)$, and third vibrational mode $\left(\Omega_{33}\right)$, and for any nonlocal parameter value, this method gives very precise results. Moreover, it is important to mention that the findings obtained by this method agree very well with those obtained by the theory developed by Aghababaei and Reddy (Higher-order shear deformation theory or TSDT) [25].

On the other hand, the first natural frequencies of the exponentially graded material (EGM) sandwich plate lying on elastic foundations, studied in the present article,
Table 3 Comparison of non-dimensional frequenciesof simply

\begin{tabular}{|c|c|c|c|c|c|}
\hline Frequencies & $\mu$ & TSDT & FSDT & Classical & Present study \\
\hline \multirow{6}{*}{$\Omega_{11}$} & 0 & 0.094 & 0.093 & 0.096 & 0.093 \\
\hline & 1 & 0.085 & 0.085 & 0.088 & 0.085 \\
\hline & 2 & 0.079 & 0.079 & 0.082 & 0.079 \\
\hline & 3 & 0.074 & 0.074 & 0.076 & 0.074 \\
\hline & 4 & 0.070 & 0.070 & 0.072 & 0.070 \\
\hline & 5 & 0.066 & 0.066 & 0.068 & 0.066 \\
\hline \multirow{6}{*}{$\Omega_{22}$} & 0 & 0.346 & 0.341 & 0.385 & 0.341 \\
\hline & 1 & 0.259 & 0.255 & 0.288 & 0.255 \\
\hline & 2 & 0.215 & 0.213 & 0.240 & 0.212 \\
\hline & 3 & 0.188 & 0.186 & 0.210 & 0.185 \\
\hline & 4 & 0.170 & 0.167 & 0.189 & 0.167 \\
\hline & 5 & 0.156 & 0.154 & 0.173 & 0.153 \\
\hline \multirow{6}{*}{$\Omega_{33}$} & 0 & 0.702 & 0.689 & 0.867 & 0.684 \\
\hline & 1 & 0.421 & 0.413 & 0.520 & 0.410 \\
\hline & 2 & 0.329 & 0.323 & 0.406 & 0.320 \\
\hline & 3 & 0.279 & 0.274 & 0.345 & 0.271 \\
\hline & 4 & 0.247 & 0.242 & 0.305 & 0.239 \\
\hline & 5 & 0.223 & 0.219 & 0.276 & 0.217 \\
\hline
\end{tabular}
supported plate between fundamental frequencies given by present model and those of Aghababaei and Reddy (TSDT) [25] $\left(E=30 \times 10^{6}, v=0.3, a=1, a / b=1, a / h=10\right)$

under different boundary conditions and for different length-to-thickness ratio values, are compared with those suggested by Sobhy [38], as displayed in Table 4. It is worth noting that the values obtained were for inhomogeneous plates ( $p=0.5$ and $p=3.5$ ) and homogeneous plates $(p=0)$ resting on elastic foundations, under any boundary conditions. It is important to mention that the results obtained are quite precise.

\section{Numerical results}

For the purpose of investigating the effect of the viscosity parameter, nonlocal parameter, damping coefficient and material properties on the vibration response of an FGM viscoelastic nanoplate (metal and ceramic: (A1 / A12O3) lying on a viscoelastic foundation, a parametric study was performed on a square plate made of the following material and having the geometric characteristics given below:

Ceramic: alumina: $E_{c}=380 \mathrm{GPa}, \rho_{c}=3800 \mathrm{~kg} / \mathrm{m}^{3}, v=0.3$ Metal: aluminum: $E_{m}=380 \mathrm{GPa}, \rho_{m}=3800 \mathrm{~kg} / \mathrm{m}^{3}, v=0.3$

Fig. 3 displays the variation of the damping ratio $\xi$ as a function of the nonlocal parameter $\mu$ for these damping coefficients of the viscoelastic foundation $C_{d}=0$ and 60 and for $K_{w}=50$ and $K_{p}=10$, with a material index $p=0,1$ and 5 under the boundary conditions (SSSS, CCSS and $C C C C$ ). Note that for the three boundary conditions and 
Table 4 Comparison of natural frequencyof EGM sandwich plate with those of Sobhy [38] $\left(b / a=2, K_{w}=50\right.$ and $\left.K_{p}=10\right)$

\begin{tabular}{|c|c|c|c|c|c|c|c|c|c|c|}
\hline \multirow{2}{*}{$\mathrm{BCs}$} & \multirow{2}{*}{ Sobhy [38] } & \multicolumn{3}{|c|}{$p=0$} & \multicolumn{3}{|c|}{$p=0.5$} & \multicolumn{3}{|c|}{$p=3.5$} \\
\hline & & $a / h=5$ & 10 & 20 & $a / h=5$ & 10 & 20 & $a / h=5$ & 10 & 20 \\
\hline \multirow{4}{*}{ SSSS } & FSDT & 1.5386 & 1.5942 & 1.6113 & 1.3971 & 1.4304 & 1.4399 & 1.3361 & 1.3589 & 1.3653 \\
\hline & TSDT & 1.5387 & 1.5947 & 1.6113 & 1.3989 & 1.4310 & 1.4401 & 1.3375 & 1.3594 & 1.3655 \\
\hline & SSDT & 1.5388 & 1.5947 & 1.6113 & 1.3990 & 1.4310 & 1.4401 & 1.3376 & 1.3594 & 1.3655 \\
\hline & Present & 1.5387 & 1.5947 & 1.6113 & 1.3989 & 1.4310 & 1.4401 & 1.3374 & 1.3594 & 1.3654 \\
\hline \multirow{5}{*}{ CCSS } & FSDT & 2.2578 & 2.5112 & 2.5997 & 1.9476 & 2.0880 & 2.1341 & 1.7472 & 1.8273 & 1.8519 \\
\hline & TSDT & 2.2590 & 2.5114 & 2.5997 & 1.9610 & 2.0930 & 2.1355 & 1.7622 & 1.8320 & 1.8532 \\
\hline & SSDT & 2.2596 & 2.5115 & 2.5998 & 1.9614 & 2.0932 & 2.1356 & 1.7630 & 1.8323 & 1.8533 \\
\hline & Present & 2.2590 & 2.5114 & 2.5997 & 1.9608 & 2.0930 & 2.1355 & 1.7617 & 1.8318 & 1.8531 \\
\hline & FSDT & 2.3359 & 2.6010 & 2.6940 & 2.0111 & 2.1586 & 2.2072 & 1.8022 & 1.8847 & 1.9107 \\
\hline \multirow{3}{*}{$\mathrm{CCCC}$} & TSDT & 2.3372 & 2.6012 & 2.6940 & 2.0250 & 2.1638 & 2.2087 & 1.8157 & 1.8895 & 1.9121 \\
\hline & SSDT & 2.3379 & 2.6013 & 2.6941 & 2.0255 & 2.1640 & 2.2088 & 1.8165 & 1.8898 & 1.9121 \\
\hline & Present & 2.3372 & 2.6012 & 2.6941 & 2.0248 & 2.1638 & 2.2087 & 1.8151 & 1.8893 & 1.9120 \\
\hline \multirow{4}{*}{ CSSS } & FSDT & 2.2176 & 2.3921 & 2.4491 & 1.9319 & 2.0302 & 2.0610 & 1.7653 & 1.8242 & 1.8421 \\
\hline & TSDT & 2.2181 & 2.3922 & 2.4493 & 1.9395 & 2.0330 & 2.0618 & 1.7720 & 1.8267 & 1.8428 \\
\hline & SSDT & 2.2185 & 2.3923 & 2.4493 & 1.9397 & 2.0331 & 2.0618 & 1.7724 & 1.8268 & 1.8428 \\
\hline & Present & 2.2182 & 2.3922 & 2.4493 & 1.9394 & 2.0329 & 2.0618 & 1.7717 & 1.8266 & 1.8428 \\
\hline \multirow{4}{*}{ CSCS } & FSDT & 2.3323 & 2.5262 & 2.5904 & 2.0265 & 2.1364 & 2.1712 & 1.8456 & 1.9117 & 1.9319 \\
\hline & TSDT & 2.3328 & 2.5262 & 2.5904 & 2.0349 & 2.1395 & 2.1720 & 1.8531 & 1.9145 & 1.9327 \\
\hline & SSDT & 2.3332 & 2.5263 & 2.5905 & 2.0352 & 2.1396 & 2.1721 & 1.8535 & 1.9146 & 1.9327 \\
\hline & Present & 2.3329 & 2.5263 & 2.5904 & 2.0348 & 2.1395 & 2.1720 & 1.8528 & 1.9144 & 1.9327 \\
\hline
\end{tabular}

for $C_{d}=0$, the damping ratio $\xi$ decreases as the nonlocal parameter $\mu$ and the index $p$ go up. For $p=0$, i.e. for an isotropic ceramic material, $\xi$ is equal to $0.0737,0.1198$ and 0.1533 , respectively, for the boundary conditions (SSSS, CCSS and CCCC) with a value of $\mu=0$. However, it is important to mention that $\xi$ decreases and can take the values $0.0525,0.0813$ and 0.1001 , respectively, when the coefficient $\mu=3$.

For $C_{d}=60$, the damping ratio $\xi$ increases proportionally with the growth of the nonlocal parameter $\mu$ and index $p$, under different boundary conditions. In general, for a value of $C_{d}$ equal to zero, there is a subcritical damping. However, for $C_{d}=60$, the damping becomes over-critical except for an isotropic plate embedded on all four sides $(C C C C)$.

Fig. 4 shows the variation of the damping ratio $\xi$ of a plate (SSSS) supported by a viscoelastic foundation $\left(C_{d}=10, K_{w}=50\right.$ and $\left.K_{p}=10\right)$ as a function of the nonlocal parameter $\mu$, for the values $0,1,5$ and 10 of the material index $p$ and damping coefficients of the viscoelastic material $\psi=0$ and 0.01 . It is noted that the damping ratio $\xi$ increases proportionally with the nonlocal parameter $\mu$, the material index $p$, and the damping coefficients of the viscoelastic material $\psi$. Here the system oscillates, and the amplitude of oscillation gradually decays to zero. In this case $(\xi<1)$, the system is called underdamped.
Moreover, the results confirm the advantage of using compositional gradient materials. It is worth noting that as the material index $p$ increases $(p=10)$, like in the case of a pure metal, the damping ratio increases too $(\xi=0.3422)$; however, it is the reverse case when the material index decreases $(p=0)$, like in the case of pure ceramics, where the damping ratio decreases $(\xi=0.2754)$, with $\mu=1$ and $\psi=0.01$, as can be seen in Fig. 4(b). Consequently, a low damping factor indicates a decreased capacity to dissipate energy.

Figs. 5, 6 and 7 illustrate the effect of the damping coefficient of the foundation on the free vibration of the FGM square nanoplate simply supported (SSSS), for different Winkler $\left(K_{w}=10,50,100,150\right)$ and Pasternak $\left(K_{p}=0,5,10\right)$ rigidities values of the foundation. In addition, the curves (a), (b) and (c) represent the variation of the imaginary frequency, real frequency and damping ratio, respectively. This variation increases progressively with the foundation parameters up to a critical point (the system goes back to its equilibrium position very quickly, without oscillating). As illustrated in Fig. 6, the vibrating system is damped for $K_{p}=5$ and $K_{w}=10$, and the damping coefficient $C_{d}$ of the foundation is equal to 27.669. However, in Fig. 7, for $K_{p}=10$, the damping coefficient $C_{d}$ of the foundation is equal to 33.085 , with $K_{w}=10$. Note that the increasing value of the damping coefficient is due to the increasing stiffness of the foundation. 

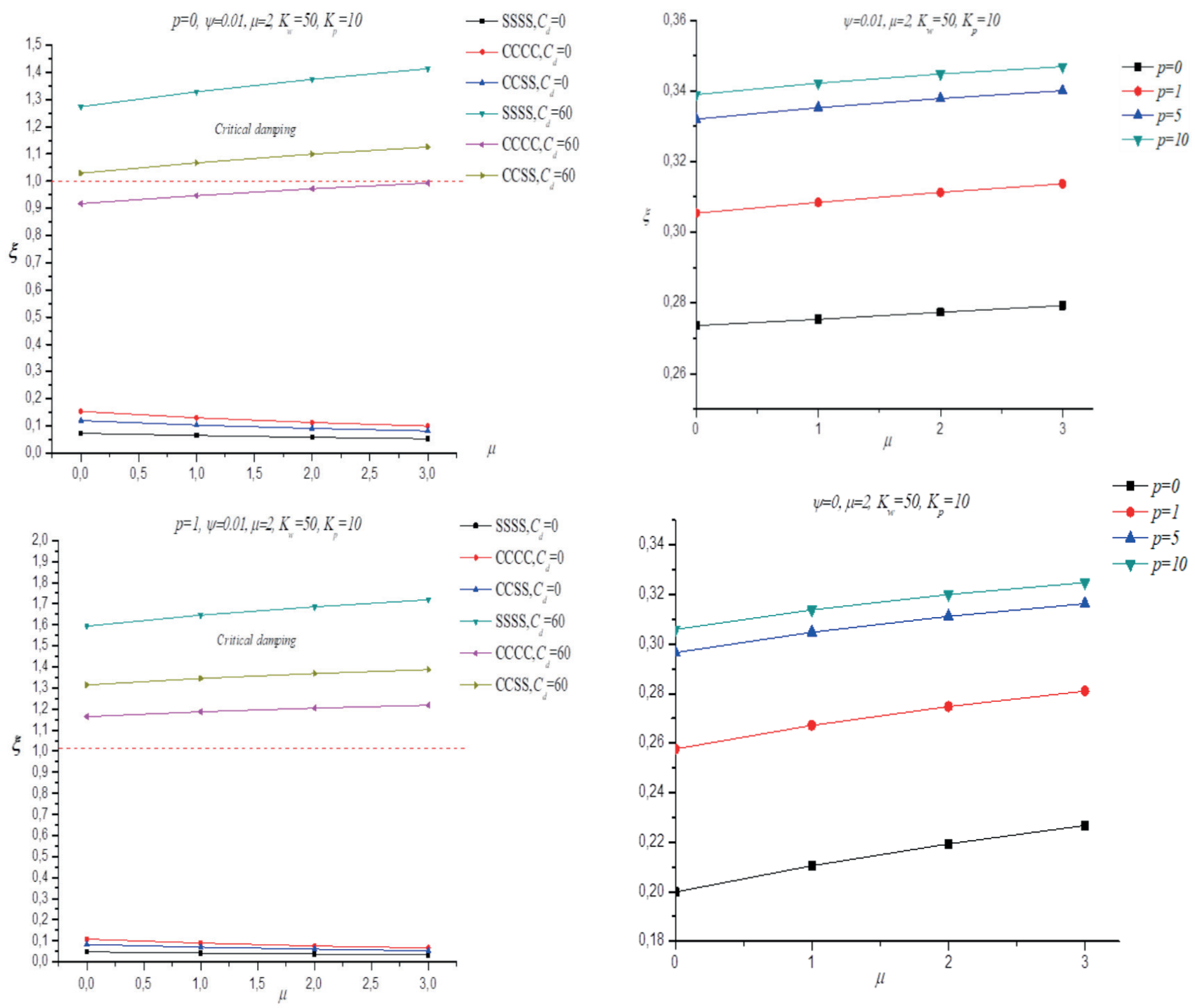

$$
\begin{aligned}
& \longrightarrow \operatorname{ssss}, C_{d}=0 \\
& \longrightarrow-\operatorname{cccC}, C_{d}=0 \\
& \longrightarrow \operatorname{ccss}, C_{d}=0 \\
& \longrightarrow \operatorname{SSss}, C_{d}=60 \\
& \longrightarrow \operatorname{cccc}, C_{d}=60 \\
& \rightarrow \operatorname{ccss}, C_{d}=60
\end{aligned}
$$

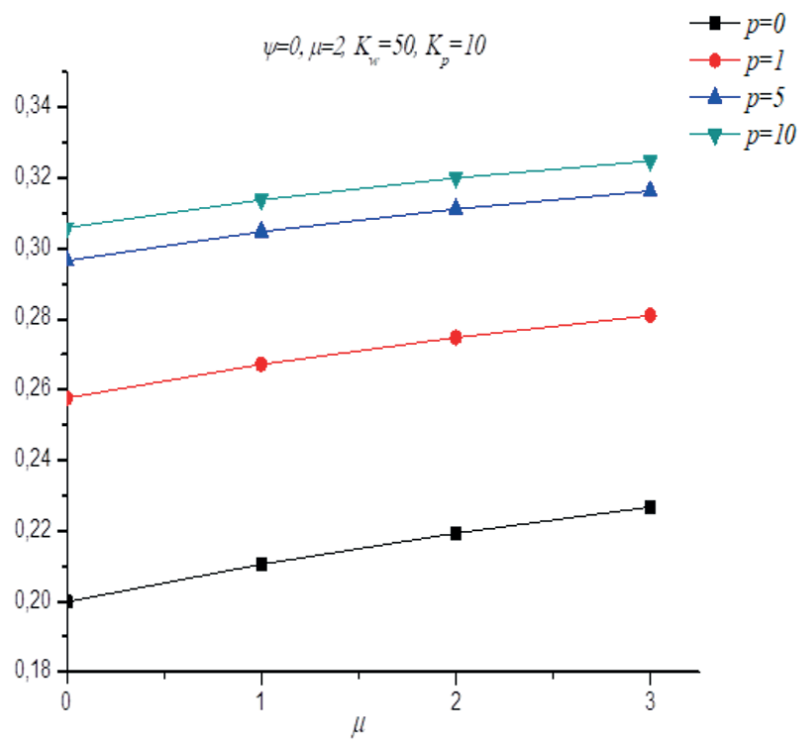

Fig. 4 Variation of the damping ratio $\xi$ of a plate (SSSS) as a function of the nonlocal parameter $\mu$ for different values of the volume fraction index $p$ and the damping coefficient $\Psi$ of the viscoelastic material

Beyond this point $(\xi>1)$, the real part of the frequency increases and admits two solutions for the same value of each of the three parameters $C_{d}, K_{p}$, and $K_{w}$, and the damping becomes over-critical; the imaginary part of the frequency tends towards zero . In this case, the plate oscillates more slowly towards the equilibrium position than it does in the case of critical damping.

Similarly, Figs. 8 and 9 show the evolution of the non-dimensional frequencies of free vibrations for different values of the nonlocal parameter $\mu$ of square FGM nanoplates simply supported SSSS with a geometric ratio $a / h$ respectively equal to 5 and 20 , with stiffnesses of the foundation $K_{w}=50$ and $K_{p}=10$, as a function of the damping coefficient of the foundation $C_{d}$. It has been observed that the real part of the non-dimensional natural frequency

Fig. 3 Variation of the damping ratio $\xi$ as a function of the nonloca parameter $\mu$, for different values of the damping coefficient $C_{d}$ of the viscoelastic foundation, of the material index $p$, under different boundary conditions 

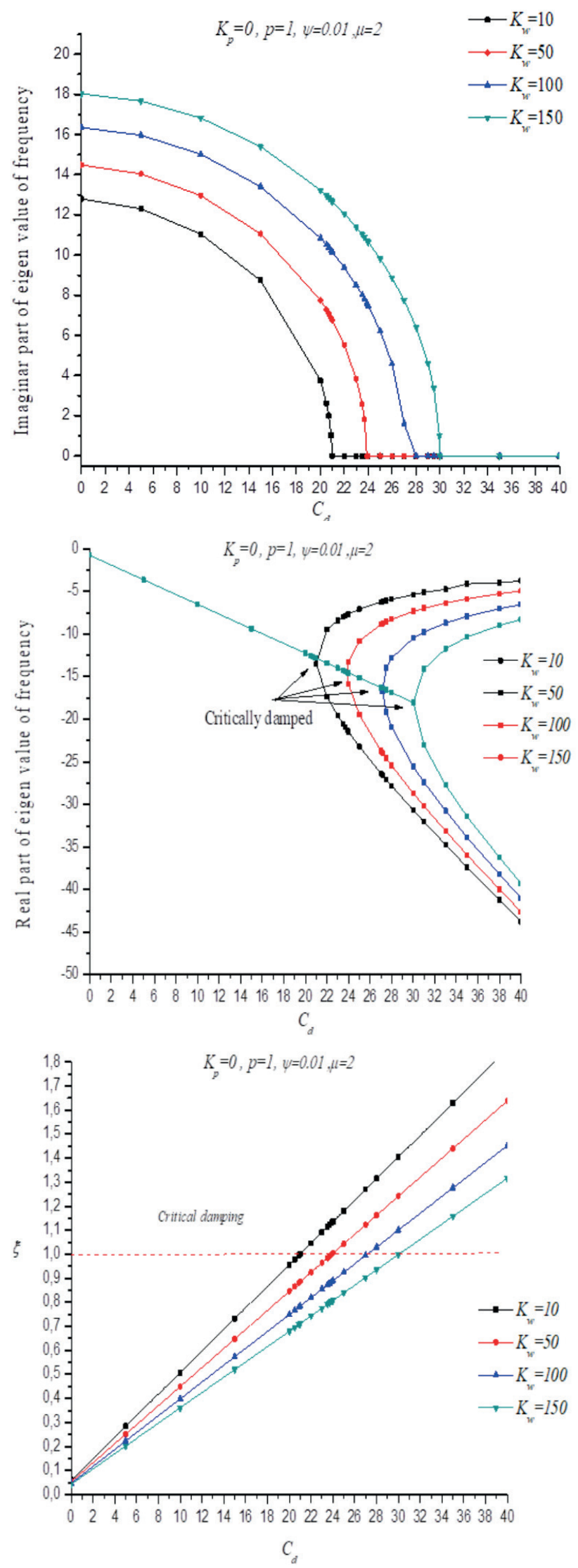

Fig. 5 Effect of the damping coefficient $C_{d}$ of the foundation on the free vibration of simply supported FGM nanoplates SSSS, for different Winkler stiffness coefficients $K_{w}$; (a) Imaginary part, (b) Real part and (c) Damping ratio
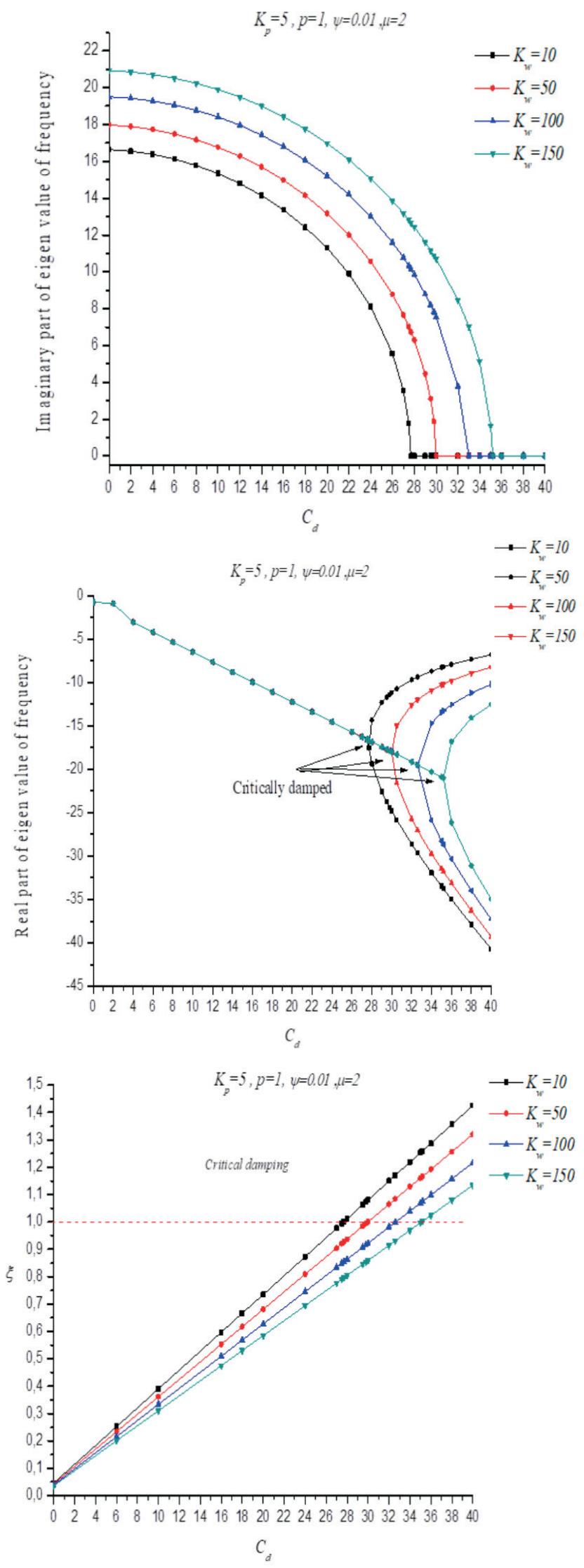

Fig. 6 Effect of the damping coefficient $C_{d}$ of the foundation on the free vibration of simply supported FGM nanoplates SSSS, for different values of the Winkler stiffness coefficient $K_{w}$; (a) Imaginary part,

(b) Real part and (c) Damping ratio 

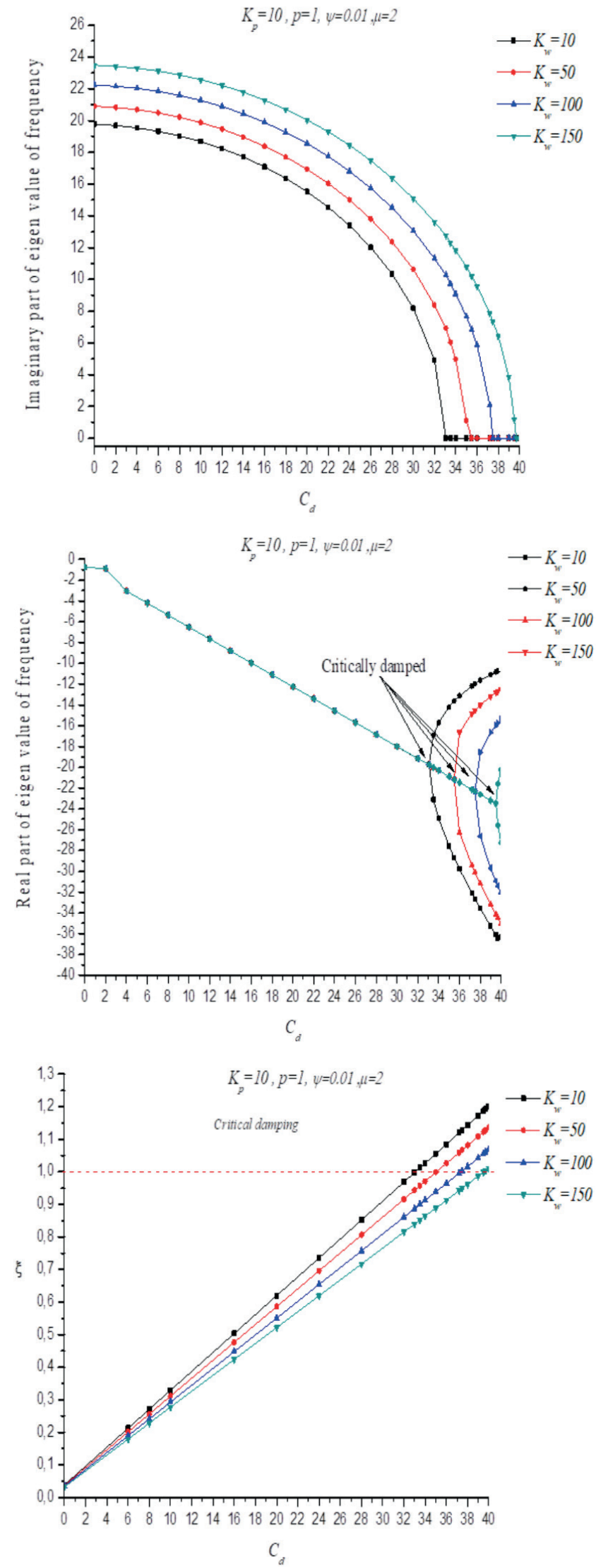

Fig. 7 Effect of the damping coefficient $C_{d}$ of the foundation on the free vibration of simply supported FGM nanoplates SSSS, for different values of the Winkler stiffness coefficient $K_{w}$; (a) Imaginary part,

(b) Real part and (c) Damping ratio increases proportionally with the nonlocal parameter $\mu$; however, the imaginary part is inversely proportional to this parameter. The additional damping reduces the nanoplate stiffness. For a geometric ratio $a / h=20$, the values of the non-dimensional frequencies are too close for the different values of the nonlocal parameter $\mu$, since the effect of the transverse deformation on these frequencies is negligible (Love-Kirchhoff theory). On the other hand, for a geometric ratio $a / h=5$ (thick plate), the effect of shearing is significant which causes an increase in the non-dimensional frequency that is represented by a significant difference between the curves as a function of $C_{d}$ up to the critical point. For example, in Fig. $8(b)$, the $(a / h=5)$ for $\mu=4$, the critical point is reached for a value of $\mathrm{Cd}$ equal to 31.646, and in Fig. 9(b) $(a / h=20)$ for $\mu=4$, the critical point is reached for a value of $C_{d}=35.845$.

Figs. 10, 11 and 12 show the effect of the damping coefficient $C_{d}$ of the foundation and the viscosity $\psi$ of the material on the non-dimensional free vibrations of simply supported square FGM nanoplates, for different volume fraction indices $p$. Curves (a), (b) and (c) respectively show the variation of the dimensionless imaginary frequency, dimensionless real frequency and damping ratio. The viscosity $\psi$ of the material induces a considerable reduction in the real and imaginary parts of the dimensionless frequencies until a critical point is reached, where the dimensionless imaginary part of the frequency becomes zero. At this point $(\xi=1)$, the nanoplate is critically damped and does not oscillate.

For example, in Fig. 10(c), for an isotropic material (ceramic), the viscosity value is $\psi=0.075$, and the critical point is reached for $C_{d}=25.75$. Note also that the critical point can be attained when $C_{d}=42.934$, for the viscosity value $\psi=0.01$. This behavior is certainly due to the decrease in the viscoelasticity of the material $\psi$.

With regard to Fig. 13, it shows the variation of the damping ratio $\xi$ as a function of the damping coefficient $C_{d}$ of the viscoelastic foundation, for an FGM nanoplate, under various boundary conditions. It is easy to notice also that for a simply supported plate $(S S S S)$, the damping is subcritical for a viscoelastic coefficient $C_{d}<35$. On the other hand, for plates under CCSS and CSSS boundary conditions, these viscoelastic coefficient values are greater and may be equal to 42 . For more rigid plates, under CSCS and $C C C C$ boundary conditions, the damping remains sub-critical for $C_{d}<48.34$. 

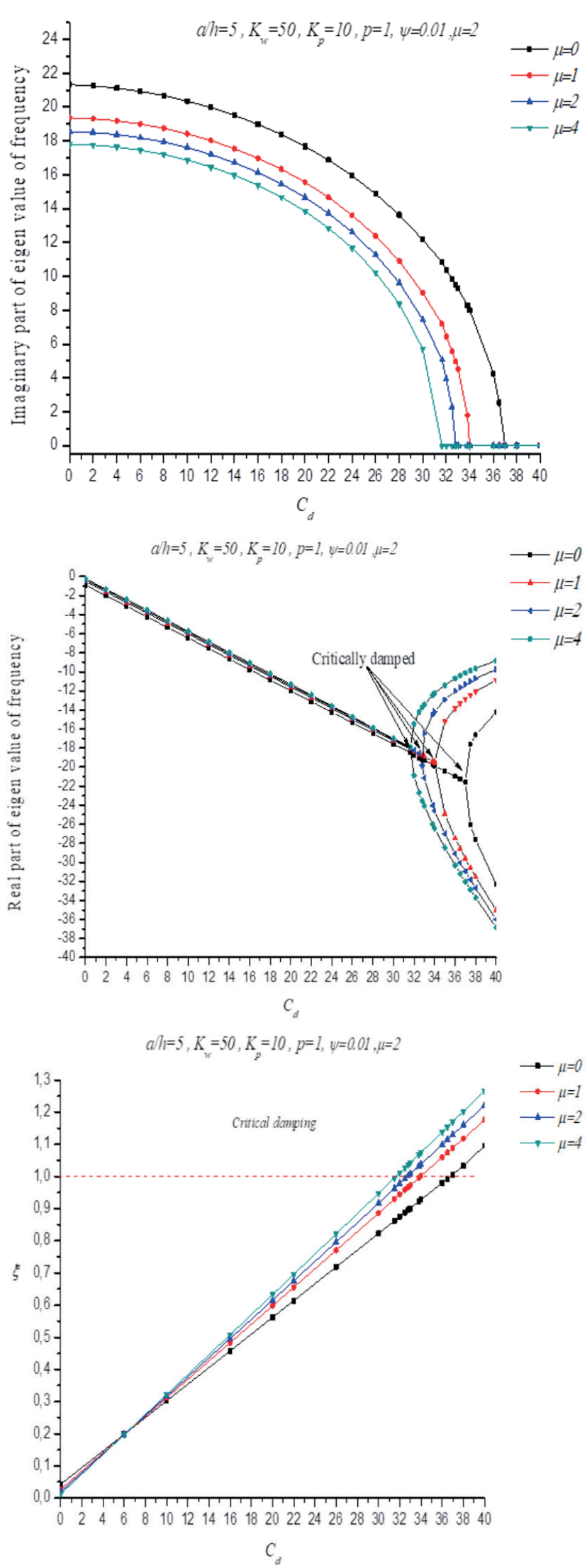

Fig. 8 Effect of the damping coefficient $C_{d}$ of the foundation on the free vibration of simply supported FGM nanoplates SSSS for different values of the nonlocal parameter $\mu$; (a) Imaginary part, (b) Real part and (c) Damping ratio
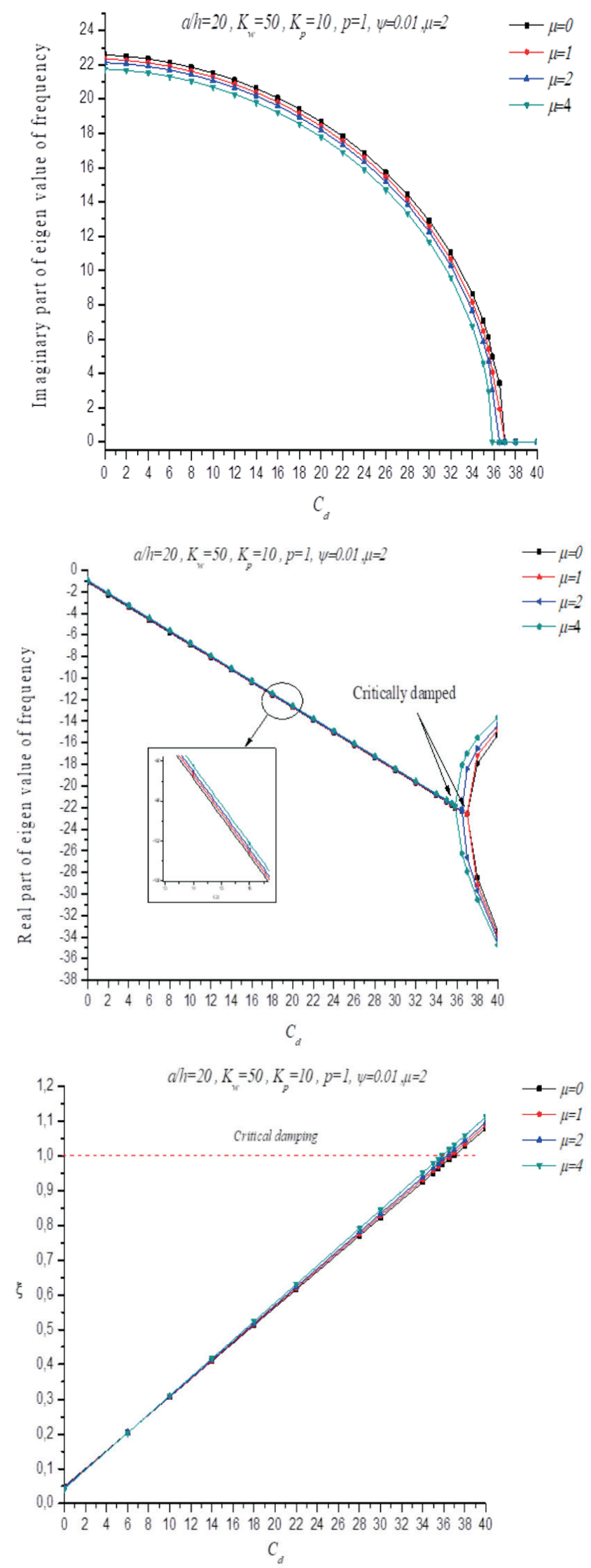

Fig. 9 Effect of the damping coefficient $C_{d}$ of the foundation on the free vibration of simply supported FGM nanoplates SSSS for different values of the nonlocal parameter $\mu$; (a) Imaginary part, (b) Real part and (c) Damping ratio 

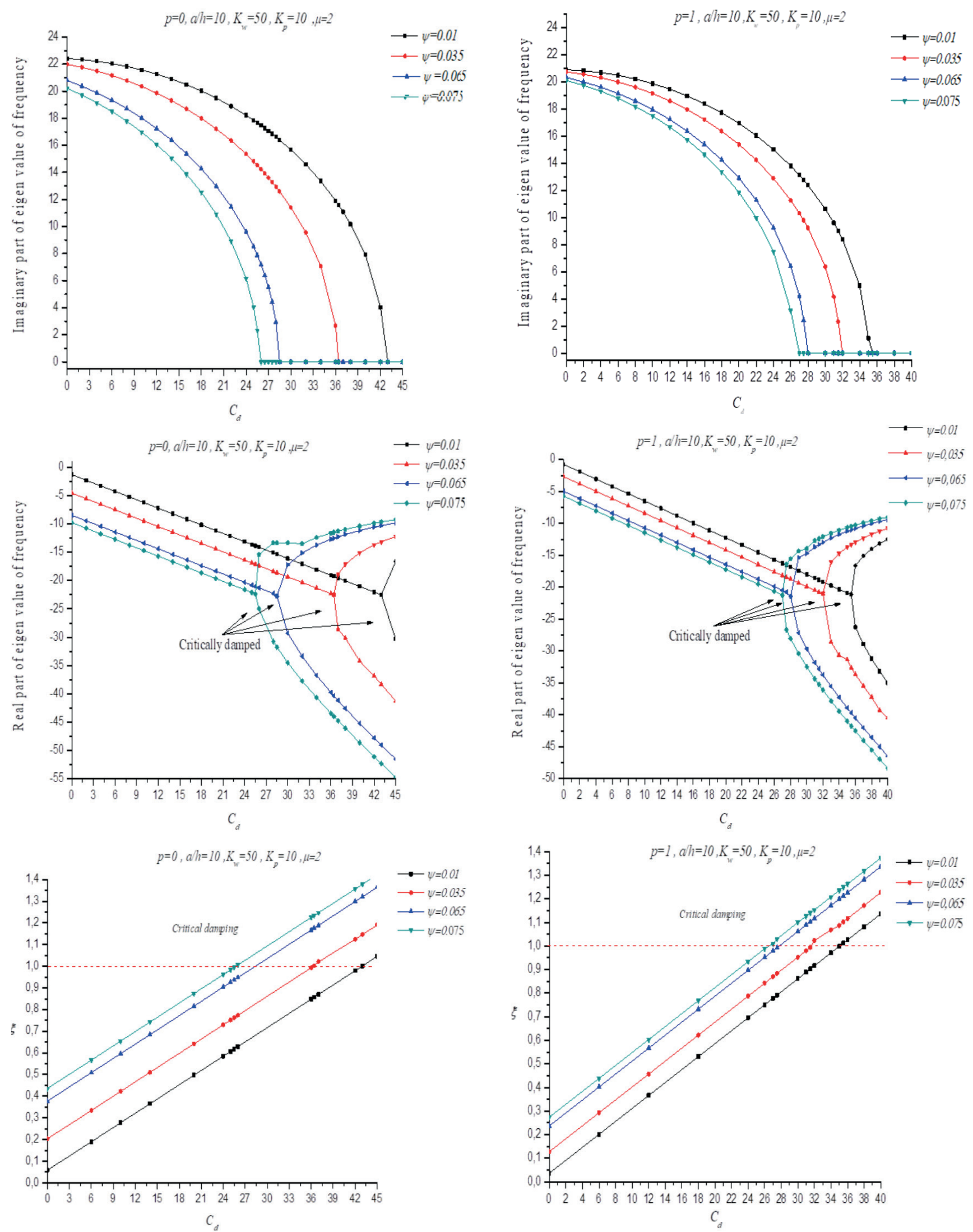

Fig. 10 Effect of the damping coefficient $C_{d}$ of the foundation on the free vibration of simply supported FGM nanoplates SSSS for different values of the viscoelastic parameter $\Psi$ of the material that constitutes the plate; (a) Imaginary part, (b) Real part and (c) Damping ratio

Fig. 11 Effect of the damping coefficient $C_{d}$ of the foundation on the free vibration of simply supported FGM nanoplates SSSS for different values of the viscoelastic parameter $\Psi$ of the material that constitutes the plate, (a) Imaginary part, (b) Real part and (c) Damping ratio 

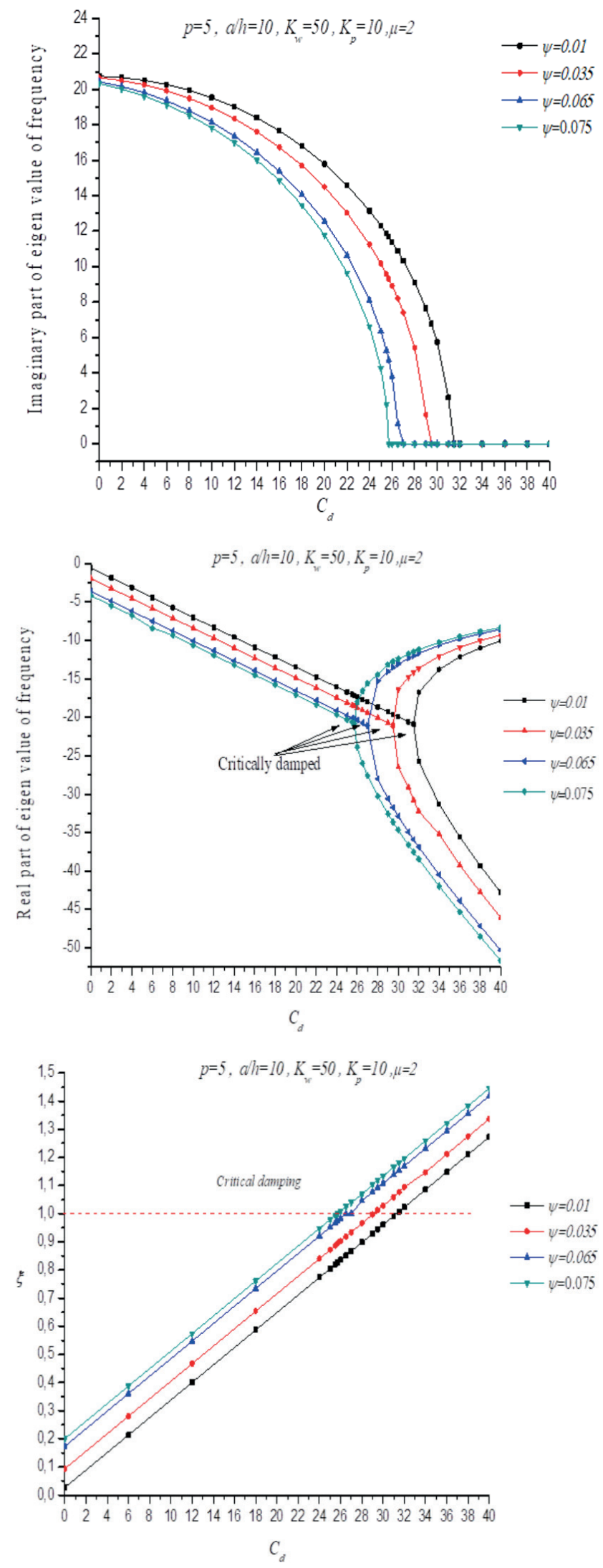

Fig. 12 Effect of the damping coefficient $C_{d}$ of the foundation on the free vibration of the simply supported FGM nanoplates SSSS, for various values of the viscoelastic parameter $\Psi$; (a) Imaginary part,

(b) Real part and (c) Damping ratio

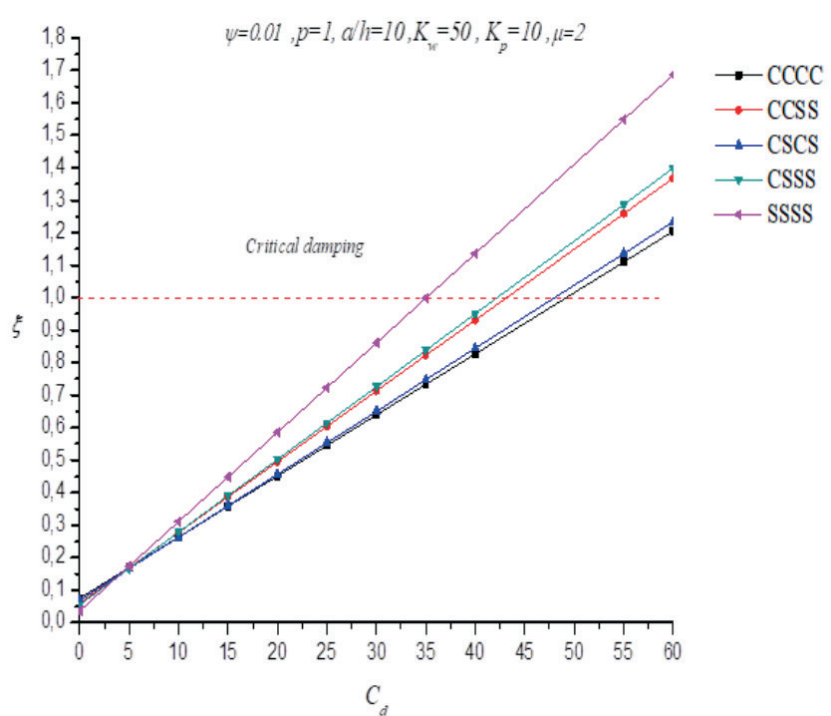

Fig. 13 Effect of the viscoelastic parameter $C_{d}$ of the foundation on the free vibration of FGM nanoplates under different boundary conditions

\section{Conclusions}

The present study aims at investigating the free vibration of an FGM viscoelastic nanoplate, lying on a viscoelastic foundation, using the Kelvin-Voigt model. A new model, which takes into account the higher-order transverse deformation theory, was utilized to develop nonlocal equilibrium equations that are based on Hamilton's principle, under various boundary conditions. The results obtained are presented for the purpose of showing the impact of different parameters on the free vibration of the FGM nanoplate.

The new model was validated based on studies previously carried out by Aghababaei and Reddy et al. [25] and Sobhy [38]. The findings in this article turned out to be in good agreement with those reported by the aforementioned authors.

The dimensionless frequency increased when the side-to-thickness ratio $(a / h)$ of the FG nanoplate went up. In addition, the effect of the transverse deformation was clearly noticed for thick nanoplates.

The vibration eigenfrequencies of simply supported FG nanoplates $(S S S S)$ were smaller than those of the embedded ones $(C C C C)$. It is interesting to note that for plates with intermediate boundary conditions (CSSS, CCSS and $C S C S$ ), the eigenfrequencies exhibited intermediate values.

The functionally graded (FG) nanoplates were better damped than the local FG plates $(\mu=0)$; this is certainly due to the fact that the nonlocal parameter reduces the structure stiffness significantly.

In the presence of an elastic foundation, the stiffness of the FG nanoplates rises, which leads to a significant increase in the vibration eigenfrequency. 
The findings of the study indicate that the predominant real part of the vibration frequency of the FGM structures is significantly influenced by the viscoelastic parameter of the material and by the damping coefficient of the foundation as well. In addition, it was found that the real part of the frequency decreases as the structural and external damping values of the foundation go down.

\section{References}

[1] Mori, T., Tanaka, K. "Average stress in matrix and average elastic energy of materials with misfitting inclusions", Acta Metallurgica, 21(5), pp. 571-574, 1973.

https://doi.org/10.1016/0001-6160(73)90064-3

[2] Yamanouchi, M., Koizumi, M, Hirai, T., Shiota, I. (eds.) "Proceedings of the First International Symposium on Functionally Gradient Materials", Gradient Materials Forum, Sendai, Japan, 1990.

[3] Koizumi, M. "The concept of FGM", In: Holt, J. B., Koizumi, M., Hirai, T., Munir, Z. A. (eds.) Ceramic Transactions, Functionally Gradient Materials, 34, American Ceramic Society, Westville, OH, USA, 1993, pp. 3-10.

[4] Suresh, S., Mortensen, A. "Fundamental of Functionally Graded Materials", IOM Communications Ltd., London, UK, 1998.

[5] Mortensen, A., Suresh, S. "Functionally graded metals and metal-ceramic composites: Part 1 processing", International Materials Reviews, 40(6), pp. 239-265, 1995.

https://doi.org/10.1179/imr.1995.40.6.239

[6] Shen, H.-S. "Functionally Graded Materials: Nonlinear Analysis of Plates and Shells", 1st ed., CRC Press, Boca Raton, FL, USA, 2011. https://doi.org/10.1201/9781420092578

[7] Kirchhoff, G. "Über das Gleichgewicht und die Bewegung einer elastischen Scheibe." (About the balance and the movement of an elastic disc), Journal fur eine und angewandte Mathematik, 1850(40), pp. 51-88, 1850. (in Deutsch) https://doi.org/10.1515/crll.1850.40.51

[8] Reissner, E. "The effect of transverse shear deformation on the bending of elastic plates", Journal of Applied Mechanics, 12, pp. 69-77, 1945.

[9] Mindlin, R. D. "Influence of rotary inertia and shear on flexural motions of isotropic, elastic plates", Journal of Applied Mechanics, 18(1), pp. 31-38, 1951.

[10] Levinson, M. "A new rectangular beam theory", Journal of Sound and Vibration, 74(1), pp. 81-87, 1981. https://doi.org/10.1016/0022-460X(81)90493-4

[11] Reddy, J. N. "A Simple Higher-Order Theory for Laminated Composite Plates", Journal of Applied Mechanics, 54(4), pp. 745752, 1984. https://doi.org/10.1115/1.3167719

[12] Touratier, M. "An efficient standard plate theory", International Journal of Engineering Science, 29(8), pp. 901-916, 1991. https://doi.org/10.1016/0020-7225(91)90165-Y
The free vibration eigenfrequencies of FG nanoplates are generally lower than those of homogeneous ceramic nanoplates. Moreover, a decrease in the power law index value ( $p=0$, pure ceramic) leads to a reduction in the damping factor, which indicates a lower capacity to dissipate energy.

The damping effects in viscoelastic material will reduce the peak values of the dynamic response, and therefore can be applied to the design to reduce the system vibration.

[13] Karama, M., Abou Harb, B., Mistou, S., Caperaa, S. "Bending, buckling and free vibration of laminated composite with a transverse shear stress continuity model", Composite Part B: Engineering, 29(3), pp. 223-234, 1998. https://doi.org/10.1016/S1359-8368(97)00024-3

[14] Aydogdu, M. "Vibration analysis of cross-ply laminated beams with general boundary conditions by Ritz method", International Journal of Mechanical Sciences, 47(11), pp. 1740-1755, 2005.

https://doi.org/10.1016/j.ijmecsci.2005.06.010

[15] Soldatos, K. P., Timarci, T. "A unified formulation of laminated composite, shear deformable, five-degrees-of-freedom cylindrical shell theories", Composite Structures, 25(1-4), pp. 165-171, 1993. https://doi.org/10.1016/0263-8223(93)90162-J

[16] Mechab, I., El Meiche, N., Bernard, F. "Analytical study for the development of a new warping function for high order beam theory", Composites Part B: Engineering, 119, pp.18-31, 2017. https://doi.org/10.1016/j.compositesb.2017.03.006

[17] Benyamina, A. B., Bouderba, B., Saoula, A. "Bending Response of Composite Material Plates with Specific Properties, Case of a Typical FGM "Ceramic/Metal" in Thermal Environments", Periodica Polytechnica Civil Engineering, 62(4), pp. 930-938, 2018. https://doi.org/10.3311/PPci.11891

[18] Shimpi, R. P., Patel, H. G. "Free vibrations of plate using two variable refined plate theory", Journal of Sound and Vibration, 296(4-5), pp. 979-999, 2006.

https://doi.org/10.1016/j.jsv.2006.03.030

[19] Shimpi, R. P., Patel, H. G. "A two variable refined plate theory for orthotropic plate analysis", International Journal of Solids and Structures, 43(22-23), pp. 6783-6799, 2006. https://doi.org/10.1016/j.ijsolstr.2006.02.007

[20] Kim, S.-E., Thai, H.-T., Lee, J. "Buckling analysis of plates using the two variable refined plate theory", Thin-Walled Structures, 47(4), pp. 455-462, 2009. https://doi.org/10.1016/j.tws.2008.08.002

[21] Thai, H.-T. , Kim, S.-E. "Free vibration of laminated composite plates using two variable refined plate theory", International Journal of Mechanical Sciences, 52(4), pp. 626-633, 2010. https://doi.org/10.1016/j.ijmecsci.2010.01.002

[22] El Meiche, N., Tounsi, A., Ziane, N., Mechab, I., Adda.Bedia, E. A. "A new hyperbolic shear deformation theory for buckling and vibration of functionally graded sandwich plate", International Journal of Mechanical Sciences, 53(4), pp. 237-247, 2011. https://doi.org/10.1016/j.ijmecsci.2011.01.004 
[23] Eringen, A. C. "Linear theory of nonlocal elasticity and dispersion of plane waves", International Journal of Engineering Science, 10(5), pp. 425-435, 1972. https://doi.org/10.1016/0020-7225(72)90050-x

[24] Eringen, A. C. "On differential equations of nonlocal elasticity and solutions of screw dislocations and surface waves", Journal of Applied Physics, 54(9), pp. 4703-4710, 1983. https://doi.org/10.1063/1.332803

[25] Aghababaei, R., Reddy, J. N. "Nonlocal third-order shear deformation plate theory with application to bending and vibration of plates", Journal of Sound and Vibration, 326(1-2), pp. 277-289, 2009. https://doi.org/10.1016/j.jsv.2009.04.044

[26] Challamel, N., Mechab, I., Elmeiche, N., Houari, M. S. A., Ameur, M., Atmane, H. A. "Buckling of Generic Higher-Order Shear Beam/Columns with Elastic Connections: Local and Nonlocal Formulation", Journal of Engineering Mechanics, 139(8), pp. 10911109, 2013. https://doi.org/10.1061/(ASCE)EM.1943-7889.0000542

[27] Zenkour, A. M., Sobhy, M. "Nonlocal elasticity theory for thermal buckling of nanoplates lying on Winkler-Pasternak elastic substrate medium", Physica E: Low-dimensional Systems and Nanostructures, 53, pp. 251-259, 2013.

https://doi.org/10.1016/j.physe.2013.04.022

[28] Farzad, E., Javad, E., Ramin, B. "Thermal buckling of FGM nanoplates subjected to linear and nonlinear varying loads on Pasternak foundation", Advances in Materials Research, 5(4) , pp. 245-261, 2016.

https://doi.org/10.12989/amr.2016.5.4.245

[29] Zenkour, A. M., Arefi, M. "Nonlocal transient electrothermomechanical vibration and bending analysis of a functionally graded piezoelectric single-layered nanosheet rest on visco-Pasternak foundation", Journal of Thermal Stresses, 40(2), pp. 167-184, 2016. https://doi.org/10.1080/01495739.2016.1229146

[30] Żur, K. K., Arefi, M., Kim, J., Reddy, J. N. "Free vibration and buckling analyses of magneto-electro-elastic FGM nanoplates based on nonlocal modified higher-order sinusoidal shear deformation theory", Composites Part B: Engineering, 182, Article number: $107601,2020$.

https://doi.org/10.1016/j.compositesb.2019.107601
[31] Wang, Y.Z., Tsai, T. J. "Static and Dynamic Analysis of a Viscoelastic Plate by the Finite Element Method", Applied Acoustics , 25(2), pp. 77-94,1988. https://doi.org/10.1016/0003-682X(88)90017-5

[32] Kiasat, M. S., Zamani, H. A., Aghdam, M. M. "On the transient response of viscoelastic beams and plates on viscoelastic medium", International Journal of Mechanical Sciences ,83, pp. 133-145, 2014.

https://doi.org/10.1016/j.ijmecsci.2014.03.007

[33] Pouresmaeeli, S., Ghavanloo, E., Fazelzadeh, S. A. "Vibration analysis of viscoelastic orthotropic nanoplates resting on viscoelastic medium", Composite Structures, 96, pp. 405-410, 2013. https://doi.org/10.1016/j.compstruct.2012.08.051

[34] Liu, J. C., Zhang, Y. Q., Fan, L. F. "Nonlocal vibration and biaxial buckling of double-viscoelastic-FGM-nanoplate system with viscoelastic Pasternak medium in between", Physics Letters A, 381(14), pp. 1228-1235, 2017. https://doi.org/10.1016/j.physleta.2017.01.056

[35] Hosseini, M., Jamalpoor, A., Bahreman, M. "Small-scale effects on the free vibrational behavior of embedded viscoelastic double-nanoplate-systems under thermal environment", Acta Astronautica, 129, pp. 400-409, 2016.

https://doi.org/10.1016/j.actaastro.2016.10.001

[36] Ebrahimi, F., Barati, M. R. "Vibration analysis of graphene sheets on viscoelastic medium incorporating hygro-thermal effects employing nonlocal strain gradient theory", Proceedings of the Institution of Mechanical Engineers, Part C: Journal of Mechanical Engineering Science, 232(13), pp. 2469-2481, 2017. https://doi.org/10.1177/0954406217720232

[37] Arefi, M., Zenkour, A. M. "Nonlocal electro-thermo-mechanical analysis of a sandwich nanoplate containing a Kelvin-Voigt viscoelastic nanoplate and two piezoelectric layers", Acta Mechanica, 228, pp. 475-493, 2017. https://doi.org/10.1007/s00707-016-1716-0

[38] Sobhy, M. "Buckling and free vibration of exponentially graded sandwich plates resting on elastic foundations under various boundary conditions", Composite Structures, 99, pp. 76-87, 2013. https://doi.org/10.1016/j.compstruct.2012.11.018 


\section{Appendix A}

$$
\begin{aligned}
& \begin{aligned}
L_{11}=\int_{0}^{a} \int_{0}^{b}+\frac{\partial X(x)}{\partial x} Y(y)\left(-I_{1} \lambda^{2} \frac{\partial X(x)}{\partial x} Y(y)+I_{1} \mu \lambda^{2} \frac{\partial X(x)}{\partial x} \frac{\partial^{2} Y(y)}{\partial y^{2}}\right. \\
+I_{1} \lambda^{2} \frac{\partial^{3} X(x)}{\partial x^{3}} Y(y)+A_{66} \frac{\partial X(x)}{\partial x} \frac{\partial^{2} Y(y)}{\partial y^{2}}+A_{66} \eta \lambda \frac{\partial X(x)}{\partial x} \frac{\partial^{2} Y(y)}{\partial y^{2}}
\end{aligned} \\
& \left.+A_{11} \frac{\partial^{3} X(x)}{\partial x^{3}} Y(y)+A_{11} \eta \lambda \frac{\partial^{3} X(x)}{\partial x^{3}} Y(y)\right) d x d y \\
& L_{12}=\int_{0}^{a} \int_{0}^{b}-\left(\frac{\partial X(x)}{\partial x}\right)^{2} Y(y) \frac{\partial^{2} Y(y)}{\partial y^{2}}\left(A_{12}+A_{66}\right)(1+\eta \lambda) d x d y \frac{\partial X(x)}{\partial x} Y(y) \\
& \begin{array}{c}
L_{13}=\int_{0}^{a b} \int_{0}^{b}\left(\left(\left(\frac{\partial^{2} Y(y)}{\partial y^{2}} \frac{\partial X(x)}{\partial x}+\frac{\partial^{3} X(x)}{\partial x^{3}} Y(y)\right) \mu-\frac{\partial X(x)}{\partial x} Y(y)\right) \mathrm{I}_{2} \lambda^{2}\right. \\
+\left(2 \mathrm{~B}_{66}+\mathrm{B}_{12}\right) \frac{\partial^{2} Y(y)}{\partial y^{2}} \frac{\partial X(x)}{\partial x}+\mathrm{B}_{11} \frac{\partial^{3} X(x)}{\partial x^{3}} Y(y)+
\end{array} \\
& \left.\left(\left(2 \mathrm{~B}_{66}+\mathrm{B}_{12}\right) \frac{\partial^{2} Y(y)}{\partial y^{2}} \frac{\partial X(x)}{\partial x}+\mathrm{B}_{11} \frac{\partial^{3} X(x)}{\partial x^{3}} Y(y)\right) \eta \lambda\right) d x d y \\
& L_{14}=\int_{0}^{a} \int_{0}^{b}-\frac{\partial X(x)}{\partial x} Y(y)\left(\left(\left(\frac{\partial^{2} Y(y)}{\partial y^{2}} \frac{\partial X(x)}{\partial x}+\frac{\partial^{3} X(x)}{\partial x^{3}} Y(y)\right) \mu-\frac{\partial X(x)}{\partial x} Y(y)\right) \mathrm{I}_{4} \lambda^{2}\right. \\
& \left.\left(2 \mathrm{~B}_{66}^{\mathrm{f}}+\mathrm{B}_{12}^{\mathrm{f}}\right) \frac{\partial^{2} Y(y)}{\partial y^{2}} \frac{\partial X(x)}{\partial x}+\mathrm{B}_{11}^{\mathrm{f}} \frac{\partial^{3} X(x)}{\partial x^{3}} Y(y)+\left(\begin{array}{c}
\left(2 \mathrm{~B}_{66}^{\mathrm{f}}+\mathrm{B}_{12}^{\mathrm{f}}\right) \frac{\partial^{2} Y(y)}{\partial y^{2}} \frac{\partial X(x)}{\partial x} \\
+\mathrm{B}_{11}^{\mathrm{f}} \frac{\partial^{3} X(x)}{\partial x^{3}} Y(y)
\end{array}\right) \eta \lambda\right) d x d y \\
& \begin{array}{c}
L_{22}=\int_{0}^{a} \int_{0}^{b}+\mathrm{A}_{22} \frac{\partial^{3} Y(y)}{\partial y^{3}} X(x)+\mathrm{A}_{66} \frac{\partial^{2} X(x)}{\partial x^{2}} \frac{\partial Y(y)}{\partial y}\left(\left(\left(\frac{\partial^{3} Y(y)}{\partial y^{3}} X(x)+\frac{\partial^{2} X(x)}{\partial x^{2}} \frac{\partial Y(y)}{\partial y}\right) \mu-X(x) \frac{\partial Y(y)}{\partial y}\right) \mathrm{I}_{1} \lambda^{2}\right.
\end{array} \\
& \left.+\left(\mathrm{A}_{22} \frac{\partial^{3} Y(y)}{\partial y^{3}} X(x)+\mathrm{A}_{66} \frac{\partial^{2} X(x)}{\partial x^{2}} \frac{\partial Y(y)}{\partial y}\right) \eta \lambda\right) d x d y \\
& L_{23}=\int_{0}^{a} \int_{0}^{b} X(x) \frac{\partial Y(y)}{\partial y}\left(\left(\left(\frac{\partial^{3} Y(y)}{\partial y^{3}} X(x)+\frac{\partial^{2} X(x)}{\partial x^{2}} \frac{\partial Y(y)}{\partial y}\right) \mu-X(x) \frac{\partial Y(y)}{\partial y}\right) \mathrm{I}_{2} \lambda^{2}\right. \\
& +\left(\mathrm{B}_{12}+2 \mathrm{~B}_{66}\right) \frac{\partial^{2} X(x)}{\partial x^{2}} \frac{\partial Y(y)}{\partial y}+\mathrm{B}_{22} \frac{\partial^{3} Y(y)}{\partial y^{3}} X(x) \\
& \left.+\left(\mathrm{B}_{22} \frac{\partial^{3} Y(y)}{\partial y^{3}} X(x)+\left(\mathrm{B}_{12}+2 \mathrm{~B}_{66}\right) \frac{\partial^{2} X(x)}{\partial x^{2}} \frac{\partial Y(y)}{\partial y}\right) \eta \lambda\right) d x d y \\
& L_{24}=\int_{0}^{a} \int_{0}^{b}-X(x) \frac{\partial Y(y)}{\partial y}\left(\left(\left(\frac{\partial^{3} Y(y)}{\partial y^{3}} X(x)+\frac{\partial^{2} X(x)}{\partial x^{2}} \frac{\partial Y(y)}{\partial y}\right) \mu-X(x) \frac{\partial Y(y)}{\partial y}\right) \mathrm{I}_{4} \lambda^{2}\right. \\
& +\left(\mathrm{B}_{12}^{\mathrm{f}}+2 \mathrm{~B}_{66}^{\mathrm{f}}\right) \frac{\partial^{2} X(x)}{\partial x^{2}} \frac{\partial Y(y)}{\partial y}+\mathrm{B}_{22}^{\mathrm{f}} \frac{\partial^{3} Y(y)}{\partial y^{3}} X(x)+ \\
& \left.\left(\mathrm{B}_{22}^{\mathrm{f}} \frac{\partial^{3} Y(y)}{\partial y^{3}} X(x)+\left(\mathrm{B}_{12}^{\mathrm{f}}+2 \mathrm{~B}_{66}^{\mathrm{f}}\right) \frac{\partial^{2} X(x)}{\partial x^{2}} \frac{\partial Y(y)}{\partial y}\right) \eta \lambda\right) d x d y
\end{aligned}
$$




$$
\begin{aligned}
& L_{33}=\int_{0}^{a} \int_{0}^{b}-X(x) Y(y)\left(\left(\begin{array}{l}
\left(\frac{\partial^{2} Y(y)}{\partial y^{2}} X(x)+\frac{\partial^{2} X(x)}{\partial x^{2}} Y(y)\right) I_{1} \\
-\left(2 \frac{\partial^{2} X(x)}{\partial x^{2}} \frac{\partial^{2} Y(y)}{\partial y^{2}}+Y(y) \frac{\partial^{4} X(x)}{\partial x^{4}}+X(x) \frac{\partial^{4} Y(y)}{\partial y^{4}}\right) I_{3}
\end{array}\right) \mu-\right. \\
& \left.\left.X(x) Y(y) I_{1}+\left(X(x) \frac{\partial^{2} Y(y)}{\partial y^{2}}+\frac{\partial^{2} X(x)}{\partial x^{2}} Y(y)\right) I_{3}\right) \lambda^{2}+\left(\begin{array}{l}
X(x) \frac{\partial^{2} Y(y)}{\partial y^{2}}+ \\
\frac{\partial^{2} X(x)}{\partial x^{2}} Y(y) \\
\mu-X(x) Y(y)
\end{array}\right)\right) \lambda \mathrm{c}_{\mathrm{d}}+ \\
& \left(\left(-2 \mathrm{D}_{12}-4 \mathrm{D}_{66}\right) \frac{\partial^{2} Y(y)}{\partial y^{2}} \frac{\partial^{2} X(x)}{\partial x^{2}}-\mathrm{D}_{11} Y(y) \frac{\partial^{4} X(x)}{\partial x^{4}}-\mathrm{D}_{22} X(x) \frac{\partial^{4} Y(y)}{\partial y^{4}}\right) \lambda \eta- \\
& \left.\mathrm{D}_{11} Y(y) \frac{\partial^{4} X(x)}{\partial x^{4}}-\mathrm{D}_{22} X(x) \frac{\partial^{4} Y(y)}{\partial y^{4}}+\left(\begin{array}{l}
-2 \frac{\partial^{2} X(x)}{\partial x^{2}} \frac{\partial^{2} Y(y)}{\partial y^{2}}-Y(y) \frac{\partial^{4} X(x)}{\partial x^{4}}- \\
X(x) \frac{\partial^{4} Y(y)}{\partial y^{4}} \\
+X(x) \frac{\partial^{2} Y(y)}{\partial y^{2}}+\frac{\partial^{2} X(x)}{\partial x^{2}} Y(y)
\end{array}\right) \mu\right) \mathrm{k}_{\mathrm{p}}+ \\
& \left.\left(\left(\frac{\partial^{2} Y(y)}{\partial y^{2}} X(x)+\frac{\partial^{2} X(x)}{\partial x^{2}} Y(y)\right) \mu-X(x) Y(y)\right) \mathrm{k}_{\mathrm{w}}+\left(-2 \mathrm{D}_{12}-4 \mathrm{D}_{66}\right) \frac{\partial^{2} Y(y)}{\partial y^{2}} \frac{\partial^{2} X(x)}{\partial x^{2}}\right) d x d y
\end{aligned}
$$

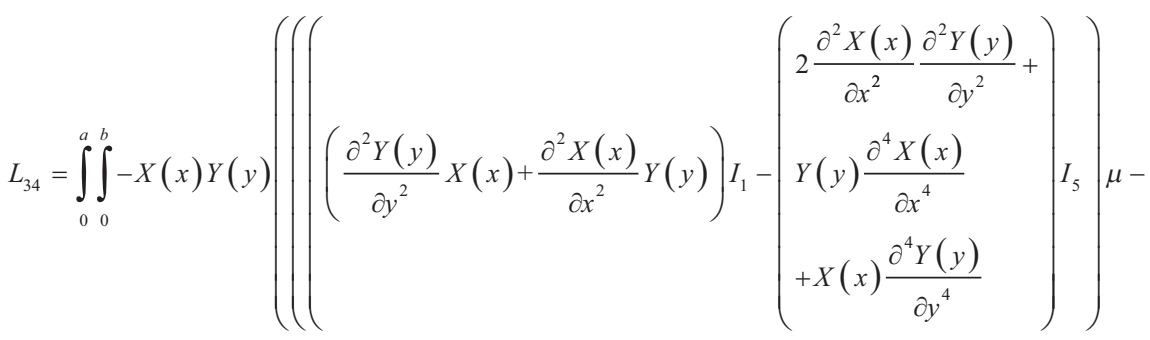

$$
\begin{aligned}
& \left.\left.X(x) Y(y) I_{1}-\left(X(x) \frac{\partial^{2} Y(y)}{\partial y^{2}}+\frac{\partial^{2} X(x)}{\partial x^{2}} Y(y)\right) I_{5}\right) \lambda^{2}+\left(\begin{array}{l}
X(x) \frac{\partial^{2} Y(y)}{\partial y^{2}}+ \\
\frac{\partial^{2} X(x)}{\partial x^{2}} Y(y) \\
-X(x) Y(y)
\end{array}\right) \mu\right) \lambda \mathrm{c}_{\mathrm{d}}+ \\
& \left(\left(2 \mathrm{D}_{12}^{\mathrm{f}}+4 \mathrm{D}_{66}^{\mathrm{f}}\right) \frac{\partial^{2} Y(y)}{\partial y^{2}} \frac{\partial^{2} X(x)}{\partial x^{2}}-\mathrm{D}_{11}^{\mathrm{f}} Y(y) \frac{\partial^{4} X(x)}{\partial x^{4}}-\mathrm{D}_{22}^{\mathrm{f}} X(x) \frac{\partial^{4} Y(y)}{\partial y^{4}}\right) \lambda \eta+\mathrm{D}_{11}^{\mathrm{f}} Y(y) \frac{\partial^{4} X(x)}{\partial x^{4}} \\
& +\mathrm{D}_{22}^{\mathrm{f}} X(x) \frac{\partial^{4} Y(y)}{\partial y^{4}}+\left(\begin{array}{l}
\left(-2 \frac{\partial^{2} X(x)}{\partial x^{2}} \frac{\partial^{2} Y(y)}{\partial y^{2}}-Y(y) \frac{\partial^{4} X(x)}{\partial x^{4}}-X(x) \frac{\partial^{4} Y(y)}{\partial y^{4}}\right) \mu+ \\
X(x) \frac{\partial^{2} Y(y)}{\partial y^{2}}+\frac{\partial^{2} X(x)}{\partial x^{2}} Y(y)
\end{array}\right) \mathrm{k}_{\mathrm{p}}+ \\
& \left.\left(\left(\frac{\partial^{2} Y(y)}{\partial y^{2}} X(x)+\frac{\partial^{2} X(x)}{\partial x^{2}} Y(y)\right) \mu-X(x) Y(y)\right) \mathrm{k}_{\mathrm{w}}+\left(2 \mathrm{D}_{12}^{\mathrm{f}}+4 \mathrm{D}_{66}^{\mathrm{f}}\right) \frac{\partial^{2} Y(y)}{\partial y^{2}} \frac{\partial^{2} X(x)}{\partial x^{2}}\right) d x d y
\end{aligned}
$$




$$
\begin{aligned}
& L_{44}=\int_{0}^{a} \int_{0}^{b}-X(x) Y(y)\left(\left(-\left(\left(\frac{\partial^{2} Y(y)}{\partial y^{2}} X(x)+\frac{\partial^{2} X(x)}{\partial x^{2}} Y(y)\right) I_{1}+\left(\begin{array}{l}
2 \frac{\partial^{2} X(x)}{\partial x^{2}} \frac{\partial^{2} Y(y)}{\partial y^{2}}+ \\
Y(y) \frac{\partial^{4} X(x)}{\partial x^{4}} \\
+X(x) \frac{\partial^{4} Y(y)}{\partial y^{4}}
\end{array}\right) I_{6}\right) \mu+\right.\right.
\end{aligned}
$$

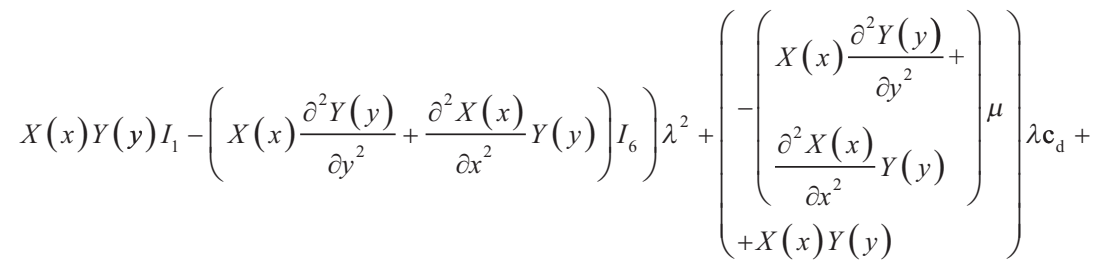

$$
\begin{aligned}
& \left(\begin{array}{l}
\left(2 \mathrm{~F}_{12}^{\mathrm{f}}+4 \mathrm{~F}_{66}^{\mathrm{f}}\right) \frac{\partial^{2} Y(y)}{\partial y^{2}} \frac{\partial^{2} X(x)}{\partial x^{2}}+\mathrm{F}_{11}^{\mathrm{f}} Y(y) \frac{\partial^{4} X(x)}{\partial x^{4}}+\mathrm{F}_{22}^{\mathrm{f}} X(x) \frac{\partial^{4} Y(y)}{\partial y^{4}}- \\
A_{44}^{f} X(x) \frac{\partial^{2} Y(y)}{\partial y^{2}}-A_{55}^{f} \frac{\partial^{2} X(x)}{\partial x^{2}} Y(y)
\end{array}\right) \lambda \eta+ \\
& \mathrm{D}_{11}^{\mathrm{f}} Y(y) \frac{\partial^{4} X(x)}{\partial x^{4}}+\mathrm{D}_{22}^{\mathrm{f}} X(x) \frac{\partial^{4} Y(y)}{\partial y^{4}}+\mathrm{F}_{11}^{\mathrm{f}} Y(y) \frac{\partial^{4} X(x)}{\partial x^{4}}+\mathrm{F}_{22}^{\mathrm{f}} X(x) \frac{\partial^{4} Y(y)}{\partial y^{4}}-
\end{aligned}
$$

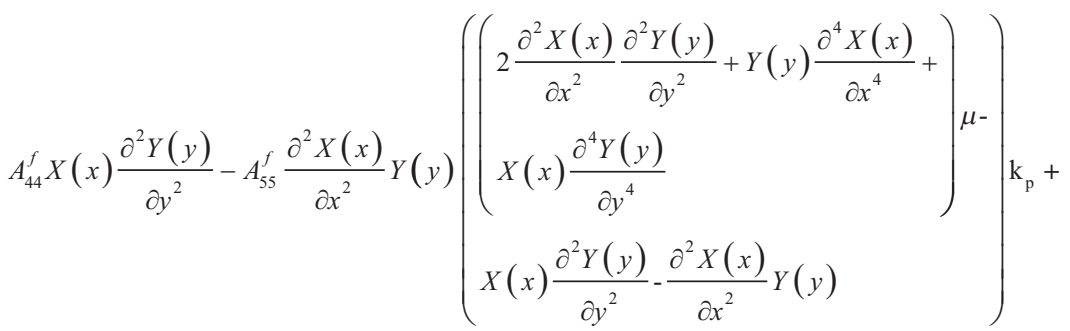

$$
\begin{aligned}
& \left.\left(X(x) Y(y)-\left(\frac{\partial^{2} Y(y)}{\partial y^{2}} X(x)+\frac{\partial^{2} X(x)}{\partial x^{2}} Y(y)\right) \mu\right) \mathrm{k}_{\mathrm{w}}+\left(2 \mathrm{~F}_{12}^{\mathrm{f}}+4 \mathrm{~F}_{66}^{\mathrm{f}}\right) \frac{\partial^{2} Y(y)}{\partial y^{2}} \frac{\partial^{2} X(x)}{\partial x^{2}}\right) d x d y
\end{aligned}
$$

